\title{
Diversidad de epífitas vasculares en árboles remanentes del género Ficus (Moraceae) en sistemas silvopastoriles del sureste de México
}

\author{
Diversity of vascular epiphytes in remnant trees of the \\ genus Ficus (Moraceae) in silvopastoral systems of \\ southeastern Mexico
}

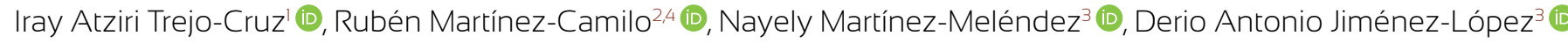

\section{Resumen}

Antecedentes y Objetivos: En los sistemas silvopastoriles es posible observar árboles remanentes que proveen de hábitats y recursos a diferentes grupos de organismos. En este estudio se analizó el rol que tienen los árboles remanentes del género Ficus (Moraceae) como refugio para albergar epífitas vasculares en la Depresión Central de Chiapas, México.

Métodos: Se evaluó la presencia de epífitas vasculares en tres sistemas silvopastoriles (dos con pastoreo extensivo y uno intensivo) y dos sitios de bosque tropical seco semiconservado. En cada sitio se seleccionaron árboles adultos de Ficus (20-30 individuos) en los cuales se muestrearon todas las epífitas vasculares (incluyendo hemiepífitas). Se realizaron análisis de varianza para determinar si hay diferencias en la riqueza y diversidad alfa (exponencial del índice de Shannon) entre los sitios, así como análisis multivariado para observar los cambios en la composición de especies.

Resultados clave: Se encontraron 77 especies, distribuidas en 36 géneros y nueve familias. Las familias más abundantes fueron Orchidaceae, Bromeliaceae y Cactaceae. En promedio, se detectaron ca. seis especies de epífitas por árbol (máximo 19). La diversidad alfa es mayor en los árboles del bosque semiconservado en comparación con los árboles remanentes presentes en los sistemas silvopastoriles. La composición de especies es heterogénea y diferente entre los sitios del bosque semiconservado y los sistemas silvopastoriles. La mitad de las especies de epífitas fueron exclusivas a un sitio determinado, principalmente en el remanente de bosque con 15 especies.

Conclusiones: Los árboles de Ficus presentan características como una corteza rugosa, ramas gruesas y casi horizontales que facilitan la colonización de epífitas y sirven como relictos de hábitat en los sistemas silvopastoriles. Por lo tanto, contribuyen al mantenimiento de la diversidad total de epífitas vasculares en paisajes altamente modificados.

Palabras clave: bosque tropical seco, Bromeliaceae, conservación, Orchidaceae, patrones de diversidad.

\section{Abstract}

Background and Aims: In silvopastoral systems, it is possible to find remnant trees that provide habitat and resources for different groups of organisms. This study analyzed the role of remnant trees of the genus Ficus (Moraceae) as refuge to harbor vascular epiphytes in the Depresión Central de Chiapas (Mexico).

Methods: The presence of vascular epiphytes in three silvopastoral systems (two with extensive and one with intensive grazing) and two sites of semi-conserved tropical deciduous forest was assessed. In each site, mature Ficus trees (20-30 individuals) were selected, and all vascular epiphytes were sampled (including hemi-epiphytes). An analysis of variance was conducted in order to determine differences in richness and alpha diversity (exponential of the Shannon index) among the sites, as well as a multivariate analysis to observe changes in species composition.

Key results: We found 77 species, distributed in 36 genera and nine families. The most abundant families were Orchidaceae, Bromeliaceae and Cactaceae. On average, ca. six epiphyte species were found per tree (maximum 19). Alpha diversity was higher in semi-conserved forests of both localities than in remnant trees present in the silvopastoral systems. Species composition is heterogeneous and different among sites of the semi-conserved forest and the silvopastoral systems. Half of the epiphyte species were exclusive to a determined site, mainly in the semi-conserved forest with 15 species.

Conclusions: Ficus trees present characteristics, such as rugose bark texture, as well as thick and almost horizontal branches, that facilitate the colonization of epiphytes, and they serve as habitat relicts in silvorpastoril systems. Therefore, they contribute to the maintenance of the total diversity of vascular epiphytes in highly modified landscapes.

Key words: Bromeliaceae, conservation, diversity patterns, Orchidaceae, tropical deciduous forest.

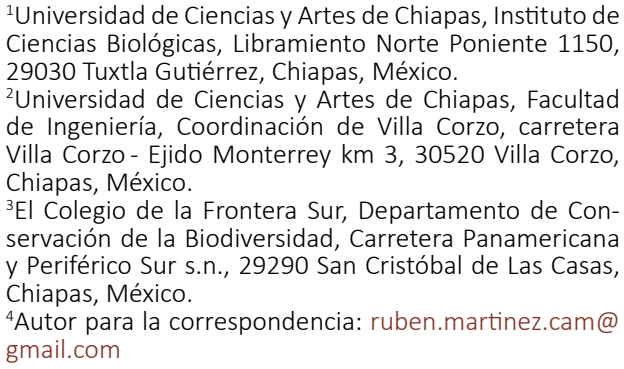
Ciencias Biológicas, Libramiento Norte Poniente 1150, 29030 Tuxtla Gutiérrez, Chiapas, México.

${ }^{2}$ Universidad de Ciencias y Artes de Chiapas, Facultad de Ingeniería, Coordinación de Villa Corzo, carretera Villa Corzo - Ejido Monterrey km 3, 30520 Villa Corzo, Chiapas, México.

${ }^{3}$ El Colegio de la Frontera Sur, Departamento de Conservación de la Biodiversidad, Carretera Panamericana y Periférico Sur s.n., 29290 San Cristóbal de Las Casas, Chiapas, México.

${ }^{4}$ Autor para la correspondencia: ruben.martinez.cam@ gmail.com

Recibido: 4 de diciembre de 2020.

Revisado: 18 de febrero de 2021.

Aceptado por Marie-Stéphanie Samain: 15 de abril de

Publicado Primero en línea: 21 de abril de 2021

Publicado: Acta Botanica Mexicana 128(2021).

Este es un artículo de acceso abierto bajo la licencia Creative Commons 4.0 Atribución-No Comercial (CC BY-NC 4.0 Internacional).
Citar como: Trejo-Cruz, I. A., R. Martínez-Camilo, N Martínez-Meléndez y D. A. Jiménez-López. 2021. Diversidad de epífitas vasculares en árboles remanentes del género Ficus (Moraceae) en sistemas silvopastoriles del sureste de México. Acta Botanica Mexicana 128: e1827. DOI: https://doi.org/10.21829/ abm128.2021.1827 


\section{Introducción}

Las epífitas vasculares (epífitas, en adelante) son un grupo de plantas que germinan y completan su ciclo de vida enraizadas de forma no parasitaria sobre otras plantas hospederas, generalmente árboles (Zotz, 2016). Los árboles hospederos proporcionan el soporte mecánico que necesitan, por lo tanto, las epífitas son dependientes de la variación espacio-temporal de los nichos ecológicos proporcionados particularmente por cada árbol hospedero (Zotz y Vollrath, 2003; Mendieta-Leiva et al., 2020). Esto puede variar en función de la ontogenia, fenología y arquitectura del árbol, pero también de su longevidad, tamaño, rugosidad de la corteza y la dinámica de la copa (Flores-Palacios y GarcíaFranco, 2006; Krömer et al., 2007a; Einzmann et al., 2015; Taylor y Burns, 2015; Wagner y Zotz, 2020). Las características de los individuos hospederos en combinación con la estratificación vertical determinan un gradiente microclimático desde el interior del sotobosque hasta la parte alta del dosel, proporcionando una diversidad de hábitats (Krömer et al., 2007b; Mendieta-Leiva et al., 2020). Incluso se ha documentado que hay una especificidad de las epífitas hacia algunos árboles hospederos, en los que encuentran condiciones de nicho ambiental adecuados y en ocasiones, únicos para su establecimiento (Nieder et al., 2001; CaIlaway et al., 2002; Zotz y Schultz, 2008; Vergara-Torres et al., 2010; Wagner et al., 2015; Wagner y Zotz, 2020).

Las epífitas contribuyen sustancialmente a la diversidad biológica de los bosques tropicales (Gentry y Dodson, 1987a; Zotz, 2013). Sin embargo, al igual que estas comunidades arbóreas, la diversidad de epífitas está constantemente amenazada debido a los diferentes procesos de pérdida y fragmentación de los bosques (Barthlott et al., 2001; Köster et al., 2009; Larrea y Werner, 2010; Krömer et al., 2014; Einzmann et al., 2016). Principalmente en aquellas regiones que presentan fuertes cambios de uso de suelo debido a las actividades productivas como la agricultura y la ganadería (Gibbs et al., 2010), estos paisajes antrópicos reciben el nombre de agroecosistemas (Ramírez-Marcial et al., 2012). Cuando la matriz de un paisaje está ocupada por sistemas de producción ganadera en donde se favorece la producción de pastos o gramíneas, de fragmentos de bosques conservados o semiconservados de diferentes tamaños y un componente leñoso (forestales o frutales), se le denomina sistema silvopastoril (Ramírez-Marcial et al., 2012; Arellano et al., 2013; Einzmann et al., 2016). En estos sistemas, a veces es posible observar la presencia de árboles que se han establecido o han persistido dentro de la matriz productiva, frecuentemente están aislados y por diversas circunstancias han quedado como árboles remanentes (Köster et al., 2009).

Los árboles remanentes son importantes porque contribuyen al mantenimiento de una porción de la diversidad de las epífitas en el paisaje (Barthlott et al., 2001; Werner et al., 2005; Hietz et al., 2006; Poltz y Zotz, 2011). Sin embargo, la configuración del sistema silvopastoril dificulta los flujos de semillas e intercambio genético de las epífitas entre los fragmentos de bosque y los árboles remanentes (Zotz y Vollrath, 2003; Flores-Palacios y García-Franco, 2006; Mondragon y Calvo-Irabien, 2006). Por ello, las comunidades de epífitas se empobrecen paulatinamente con el tiempo de aislamiento y con el aumento de la distancia hacia los remanentes de los bosques (Werner y Gradstein, 2008; Köster et al., 2009; Poltz y Zotz, 2011; Werner, 2011; Einzmann et al., 2016). El aislamiento de los árboles remanentes provoca cambios en el microclima (hacia niveles más altos de luz y estrés por desecación asociados con la alteración del bosque) y restricciones de dispersión (FloresPalacios y García-Franco, 2008; Werner y Gradstein, 2008; Larrea y Werner, 2010). Sin embargo, los efectos del aislamiento sobre la diversidad de epífitas aún son poco comprendidos, aunque se sabe de una disminución del reclutamiento de individuos juveniles y aumentos en la mortalidad con el tiempo (Larrea y Werner, 2010; Werner, 2011).

Los bosques tropicales secos tienen una gran diversidad de epífitas, aunque no en las mismas proporciones que sus contrapartes más húmedas y de mayor precipitación (Gentry y Dodson, 1987b; Kreft et al., 2004). En estas comunidades arbóreas se presentan fuertes presiones ambientales sobre las epífitas, principalmente una baja tasa de precipitación, menor cantidad de humedad atmosférica y niveles elevados de radiación solar (Gentry y Dodson, 1987b; Vergara-Torres et al., 2010). En respuesta, muchas especies de epífitas han desarrollado mecanismos de tolerancia a una alta incidencia solar y la menor disponibilidad de agua y humedad, como por ejemplo una mayor cantidad de tricomas, estomas hundidos, órganos especializa- 
dos para almacenar el agua (pseudobulbos en orquídeas), hojas arrosetadas como un tanque en las bromeliáceas, suculencia, poiquilohidria y la asimilación de $\mathrm{CO}_{2}$ por medio del metabolismo ácido de las crasuláceas (Zotz y Andrade, 2001; Werner et al., 2005; Hietz, 2010; Cach-Pérez et al., 2013; Chilpa-Galván et al., 2013, 2017). Algunas familias de plantas vasculares con muchas especies de epífitas que presentan estas adaptaciones y que tienen una alta diversidad en los bosques secos son Bromeliaceae, Cactaceae y Orchidaceae (Gentry y Dodson, 1987a; Vergara-Torres et al., 2010).

En el sureste de México, en la región conocida como Depresión Central de Chiapas, se presentaba una de las mayores extensiones de bosque tropical seco del país (Vaca, 2012). Actualmente, tiene menos de $10 \%$ de la cobertura original de bosque y el resto se trata de áreas dedicadas a la actividad agrícola, al pastoreo de ganado vacuno y una gran cantidad de localidades urbanas y rurales (Vaca, 2012). Una dinámica similar fue detectada para otras regiones del Neotrópico en donde se presenta este tipo de vegetación (Graesser et al., 2015; Armenteras et al., 2017). En la Depresión Central de Chiapas son frecuentes los sistemas silvopastoriles en donde sobresalen árboles remanentes del género Ficus L. (Moraceae). Estos son multipropósito en la actividad ganadera, ya que son usados como fuente de sombra, leña o madera y sus frutos y follaje como alimento para el ganado vacuno (Guevara et al., 1992; Serio-Silva et al., 2002; Benhin, 2006). Algunos estudios han evaluado la importancia de los árboles de Ficus por su contribución en la diversidad, estructura y el funcionamiento de algunas comunidades de animales, como la provisión de alimentos para las aves (Piedra-Malagón et al., 2006), murciélagos (Galindo-González et al., 2000) y monos (Serio-Silva et al., 2002). Sin embargo, se conoce poco sobre el rol de los árboles remanentes de Ficus como refugio para albergar epífitas en los sistemas silvopastoriles (Gonçalves y Waechter, 2002).

El objetivo de este trabajo fue analizar la variación de la diversidad de las epífitas entre los árboles remanentes de Ficus en sistemas silvopastoriles y de aquellos ubicados en fragmentos de bosque semiconservado. Nuestra hipótesis de trabajo es que la riqueza y diversidad alfa de epífitas en árboles remanentes de Ficus serán inferiores en los sistemas silvopastoriles en comparación a los detectados en los árboles dentro de los fragmentos de bosques semiconservados. La menor diversidad de epífitas en los árboles remanentes se debe a una combinación en la limitación de los procesos de dispersión de corto alcance y a las restricciones microclimáticas impuestas por la fragmentación del bosque, que incluyen mayores niveles de luz $y$, consecuentemente, menor humedad. Debido a los cambios ambientales también se esperan cambios en la composición de especies de las epífitas. No obstante, sugerimos que los árboles remanentes de Ficus en los sistemas silvopastoriles tienen un papel determinante como relictos de hábitat para garantizar el mantenimiento inclusive de una proporción importante de epífitas.

\section{Materiales y Métodos}

\section{Área de estudio}

La Depresión Central de Chiapas en el sureste de México ha tenido una pérdida significativa de la cobertura arbórea de bosque tropical seco y actualmente se observan paisajes con una matriz dominada por áreas destinadas a la agricultura, pastizales o potreros, asentamientos humanos, fragmentos de bosques y bosques secundarios o acahuales (Ramírez-Marcial et al., 2012; Vaca, 2012; Arellano et al., 2013; Gordillo-Ruiz y Castillo-Santiago, 2017).

Los acahuales generalmente son de porte bajo, con árboles que miden de 4 a $6 \mathrm{~m}$ de altura (p.ej., diferentes especies del género Acacia Mill., Fabaceae) y que tienen una presencia importante de plantas arbustivas (Arellano et al., 2013). En los sistemas silvopastoriles destaca el uso de algunas especies arbóreas, principalmente Acacia pennatula (Schltdl. \& Cham.) Benth. y diferentes especies del género Ficus, aunque no se sabe con certeza si es deliberado o simplemente es el resultado de una baja intensidad de pastoreo en condiciones de un sistema silvopastoril no intensivo (Ramírez-Marcial et al., 2012).

En el área de estudio, de la vegetación original solo quedan algunos fragmentos de bosques en estado semiconservado debido a la fuerte extracción de leña y madera. Estos se caracterizan por la presencia de especies de árboles que en general no superan $10 \mathrm{~m}$ de altura, como Bursera simaruba (L.) Sarg., Cochlospermum vitifolium (Willd.) Spreng., Cordia alliodora (Ruiz \& Pav.) Oken, Ficus spp., 
Luehea candida (DC.) Mart. y Lysiloma acapulcense (Kunth) Benth. (Rocha-Loredo et al., 2010; Pineda-Diez de Bonilla et al., 2012). En estos paisajes predominan los sistemas silvopastoriles; incluso, recientemente, se han incrementado las superficies destinadas a este sistema de producción de ganado (Gordillo-Ruiz y Castillo-Santiago, 2017).

\section{Sitios de muestreo}

Se ubicaron dos localidades de estudio que se denominaron Piedra Parada (PP) y San Fernando (SF) (Fig. 1). Ambas se caracterizan por la presencia de terrenos planos y lomeríos suaves, con suelos generalmente delgados y con afloramiento rocoso. El clima predominante corresponde al cálido subhúmedo con lluvias en verano, temperatura promedio anual de 24 a $33{ }^{\circ} \mathrm{C}$ y precipitación anual promedio de ca. $800 \mathrm{~mm}$; el periodo de secas dura aproximadamente siete meses (diciembre-junio) (Arellano et al., 2008). El estudio se realizó en una estrecha amplitud altitudinal (mínimo $=790 \mathrm{~m}$, máximo $=1019 \mathrm{~m}$, promedio $=900$, desviación estándar=72) en ambas localidades para evitar un efecto de la variación ambiental (p.ej., temperatura y humedad) impuesta por los cambios altitudinales, que suelen ser particularmente relevantes sobre la distribución de la diversidad de epífitas (Krömer et al., 2013; Jiménez-López et al., 2020).
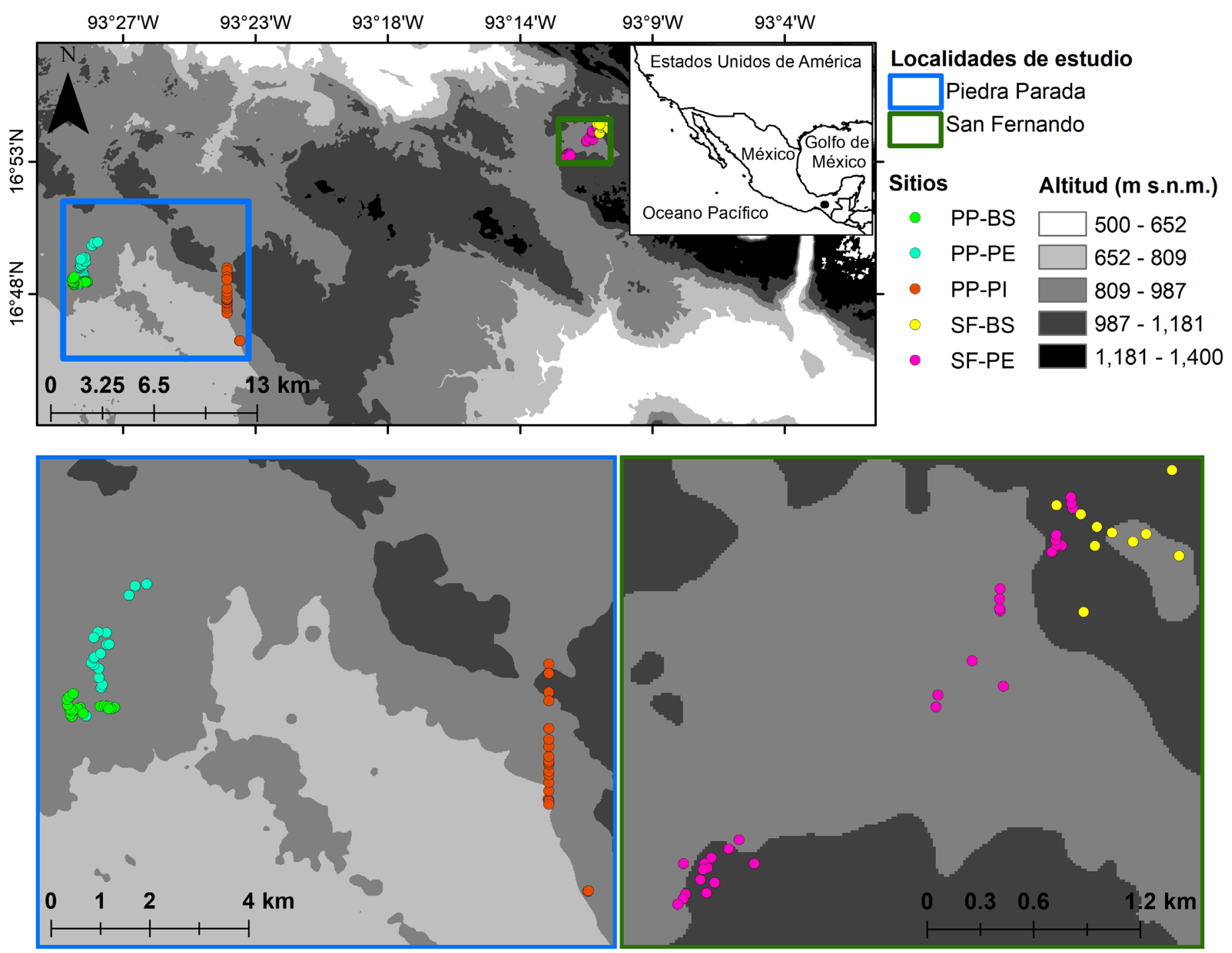

Figura 1: Área de estudio y ubicación de los cinco sitios de muestreo (SF-BS=San Fernando-bosque semiconservado, SF-PE=San Fernando-pastoreo extensivo, PP-BS=Piedra Parada-bosque semiconservado, PP-PE=Piedra Parada-pastoreo extensivo, PP-PI=Piedra Parada-pastoreo intensivo) en la Depresión Central de Chiapas, México. 


\section{Diseño de muestreo y selección de árboles}

Se definieron dos tipos de sistemas silvopastoriles, el pastoreo intensivo y el extensivo, y fragmentos de bosque semiconservado, lo que resultó en una combinación de cinco sitios (Fig. 1): (1) San Fernando-bosque semiconservado (SF-BS), (2) San Fernando-pastoreo extensivo (SF-PE), (3) Piedra Parada-bosque semiconservado (PP-BS), (4) Piedra Parada-pastoreo extensivo (PP-PE), (5) Piedra Parada-pastoreo intensivo (PP-PI).

En el pastoreo extensivo no hay un sistema tecnificado en la siembra de pastos perennes, pues los animales pastorean de manera libre y por periodos prolongados, y generalmente hay una cantidad considerable de plantas herbáceas, arbustivas y arbóreas (Soto-Pinto et al., 2008). En el pastoreo intensivo hay un sistema tecnificado de los pastos perennes (con sistemas de riego y barbecho), principalmente de la especie introducida Cynodon nlemfuensis Vanderyst (pasto estrella), donde la cobertura arbórea es eliminada casi en su totalidad y no se permite que crezcan otras plantas arbustivas y/o herbáceas distintas del pasto (Soto-Pinto et al., 2008). Sin embargo, no se encontró un área con esta descripción de pastoreo intensivo en la localidad San Fernando.

Se seleccionaron árboles hospederos adultos del género Ficus pertenecientes a tres especies: F. aurea Nutt, F. maxima Mill y F. petiolaris Kunth. Inicialmente se planteó seleccionar 30 individuos en cada sitio; sin embargo, debido al nivel de antropización y heterogeneidad solo se muestrearon 20, excepto en SF-PE en donde fue posible encontrar los 30 árboles. La selección tuvo dos criterios, que tuvieran un diámetro a la altura del pecho (DAP) $>20 \mathrm{~cm}$ y que entre ellos tuvieran una separación $>50 \mathrm{~m}$.

En las condiciones con pastoreo (intensivo y extensivo) el criterio de selección fue que los árboles estuvieran distribuidos en toda el área de pastoreo, considerando individuos cercanos al fragmento del bosque como aquellos ubicados en el centro. Sin embargo, la configuración del paisaje es muy heterogénea y existen más de un fragmento de bosque semiconservado. Cada árbol fue georreferenciado con un GPS Garmin 64s (Garmin Ltd., Olathe, Kansas, EUA) para obtener las coordenadas geográficas (latitud y longitud) y la altitud (m s.n.m.).

\section{Muestreo de epífitas}

En cada árbol hospedero se registró el número de especies y la abundancia de holoepífitas (plantas que germinan y se establecen en los árboles sin tener contacto con el suelo) y hemiepífitas primarias (plantas que germinan e inician sus primeras etapas de crecimiento sobre otras plantas y luego extienden sus raíces al suelo) (Zotz, 2016). El muestreo se realizó desde la base del árbol hasta el dosel. Las observaciones se hicieron con el apoyo de binoculares y solo en algunos casos fue necesario trepar a los árboles (sin equipo de escalamiento), ya que en general estos son de porte bajo ( $<10 \mathrm{~m}$ ) y no hay muchas dificultades para observar epífitas.

No se contabilizaron los individuos en etapa de plántulas, debido a la dificultad para identificarlas a nivel de especie y por las altas tasas de mortalidad que presentan (Winkler et al., 2005). Para las epífitas gregarias, es decir, aquellas que presentan reproducción asexual y forman colonias (stand), se contabilizó cada una como un individuo por su similitud genética (Zotz y Schultz, 2008).

Las epífitas se identificaron en campo a nivel de especie. Cuando no fue posible identificarlas se les asignó una morfoespecie y se obtuvo una muestra botánica para su posterior determinación taxonómica con ayuda de literatura especializada (p.ej., Mickel y Smith, 2004; Hágsater et al., 2005; Beutelspacher-Baigts, 2013) y mediante comparación con ejemplares depositados en el Herbario Eizi Matuda (HEM) de la Universidad de Ciencias y Artes de Chiapas. Los ejemplares fueron depositados en el herbario HEM.

\section{Análisis}

Se construyeron curvas de acumulación de especies para visualizar el esfuerzo de muestreo general y para cada sitio de estudio. Se calculó la diversidad alfa con el índice de Shannon (exponencial) bajo el enfoque de diversidad verdadera o números de Hill, que considera a las abundancias relativas de las especies como equivalentes (Jost, 2006). Para comparar los promedios de la riqueza y diversidad alfa de las epífitas, así como el diámetro de los árboles hospederos entre los cinco sitios, se realizaron análisis de varianza de una vía (ANOVA). Si el modelo de ANOVA resultó significativo, entonces se realizaron pruebas pareadas de Tukey para determinar las combinaciones que fueron diferentes. 
Se ajustaron modelos de regresión simple para observar la relación entre el DAP y la diversidad alfa de epífitas. Para observar la variación en la composición de las epífitas, se realizó un análisis de escalamiento multidimensional no métrico (Non-metric multidimensional scaling, NMDS), que es una técnica multivariante para observar visualmente las distancias entre los conjuntos de datos (especies y unidades de muestreo). Para evaluar las relaciones entre las especies y el efecto de las condiciones, se realizó un análisis de varianza usando matrices de distancia y prueba de permutación con pseudo- $F$ radios (PERMANOVA) con la función "Adonis" de la librería Vegan (Oksanen et al., 2019). Se verificó la correcta normalidad de los residuales en los modelos de los análisis de varianza y de regresión simple. En ausencia de la normalidad de los residuales se aplicaron transformaciones al logaritmo natural de las variables explicativas para normalizarlos. En todos los análisis se usó un valor de referencia de alfa de $p<0.05$. Las curvas de acumulación de especies y los valores de diversidad alfa se obtuvieron con la librería Vegan (Oksanen et al., 2019). Todos los análisis se realizaron con el software R ( R Core Team, 2020).

\section{Resultados}

\section{Composición florística}

Se encontraron 77 especies de epífitas en 110 árboles (Fig. 2A), que corresponden a 36 géneros y nueve familias (Apéndice). Se asignaron nueve morfoespecies ya que su determinación a nivel específico no fue posible por la falta de estructuras reproductivas. El sitio SF-BS fue el más importante por el número de especies con 45 , y la de menor fue PP-PI con 16 (Fig. 2B). La familia con mayor riqueza específica fue Orchidaceae con 27, le siguen en orden de importancia Bromeliaceae (22) y Cactaceae (9); estas tres familias agrupan $75 \%$ del total. A nivel de género, el más importante fue Tillandsia L. con 16 especies, muy por encima de Peperomia Ruiz \& Pav. y Polypodium L. (seis cada uno), y de los géneros de Orchidaceae, Epidendrum L. y Trichocentrum Poepp. \& Endl. (cuatro cada uno).

Del total de especies, tres fueron asignadas como hemiepífitas (Clusia sp. 1, Oreopanax guatemalensis (Lem. ex Bosse) Decne. \& Planch. y Philodendron radiatum Schott) y el resto como holoepífitas (Apéndice). Las epífitas más abundantes fueron las bromelias del género Tillandsia: T. zoquensis Ehlers con 2589 individuos, T. schiedeana Steud. con 1212 y T. belloensis W. Weber con 992. Esto contrasta con un número importante de especies (ca. $20 \%$ del total) para las cuales solo se encontraron uno (11 especies) o dos individuos (seis), singletons y doubletons, respectivamente.

La contribución relativa de las familias y su riqueza varía dependiendo del sitio (Fig. 3). Orchidaceae contribuyó con un mayor número de especies a nivel general y en SF-BS y PP-PE. Bromeliaceae tuvo mayor riqueza en SF-PE y PP-PI y PP-BS. Únicamente en PP-BS se observó que la familia Bromeliaceae contribuyó con más de la mitad de las especies (65\%) a la riqueza total. En general, todas las demás familias contribuyeron con menos de $15 \%$ a la riqueza total en cada sitio, aunque las siguientes familias estuvieron presentes en todos: Araceae, Cactaceae, Piperaceae y Polypodiaceae (Fig. 3).

\section{Patrones de diversidad}

Se encontraron en promedio 5.7 especies (desviación estándar $(D E)=3.1$, mínimo $(M i n)=2$, máximo $(M a x)=19)$ de epífitas en los árboles remanentes de Ficus. El promedio general de diversidad alfa fue de 3.6 especies ( $D E=1.5$, Min=1.1, Max=10.7). Los árboles con la mayor riqueza de epífitas fueron muestreados en SF-BS con 19, 16 y 15 especies. En SF-BS se detectaron los promedios más elevados de riqueza (9.1 especies) y de diversidad (5.2 especies) por árbol remanente, y estos valores fueron estadísticamente diferentes de los obtenidos para los otros sitios $(p>0.05$; Cuadro 1). No se encontraron diferencias significativas en los promedios de diversidad entre las demás combinaciones de sitios ( $p>0.05$, en todos los casos). El sitio PP-PI tuvo el valor más bajo en riqueza (3.4) y fue significativamente distinto estadísticamente de los valores de los sitios SF-PE y PP-BS.

Sobre la variación en la composición de las epífitas, se encontró que cerca de la mitad (52\%) de las especies muestreadas fueron exclusivas o solo se encontraron en un sitio (Fig. 4). En SF-BS fueron exclusivas 15 (18\% del total) y en SF-PE y PP-PE nueve en cada uno (11\% en ambos). Las epífitas que estuvieron en todos los sitios fueron Tillandsia fasciculata Sw., T. schiedeana, T. recurvata (L.) L. y T. zoquensis; las que estuvieron en cuatro fueron Catopsis 


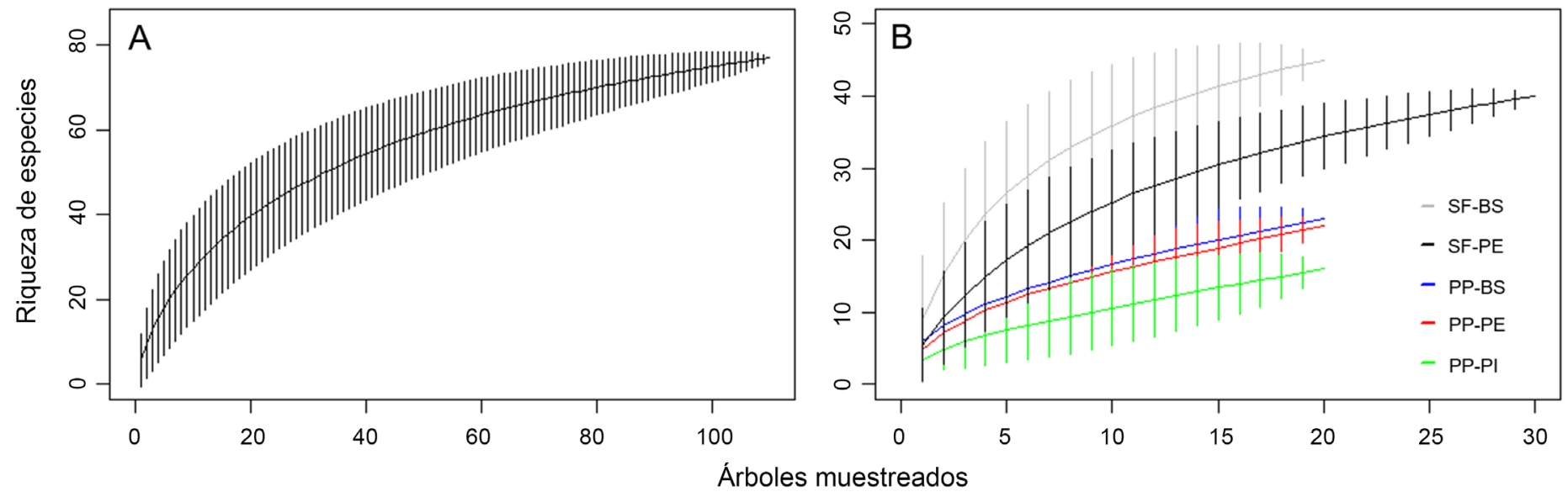

Figura 2: Curvas de acumulación de especies de epífitas vasculares en 110 árboles remanentes del género Ficus L. ubicados en cinco sitios en la Depresión Central de Chiapas, México. Las barras indican el intervalo de confianza a 95\%. A. todas las especies; B. para cada sitio. SF-BS=San Fernando-bosque semiconservado, SF-PE=San Fernando-pastoreo extensivo, PP-BS=Piedra Parada-bosque semiconservado, PP-PE=Piedra Paradapastoreo extensivo, PP-PI=Piedra Parada-pastoreo intensivo.

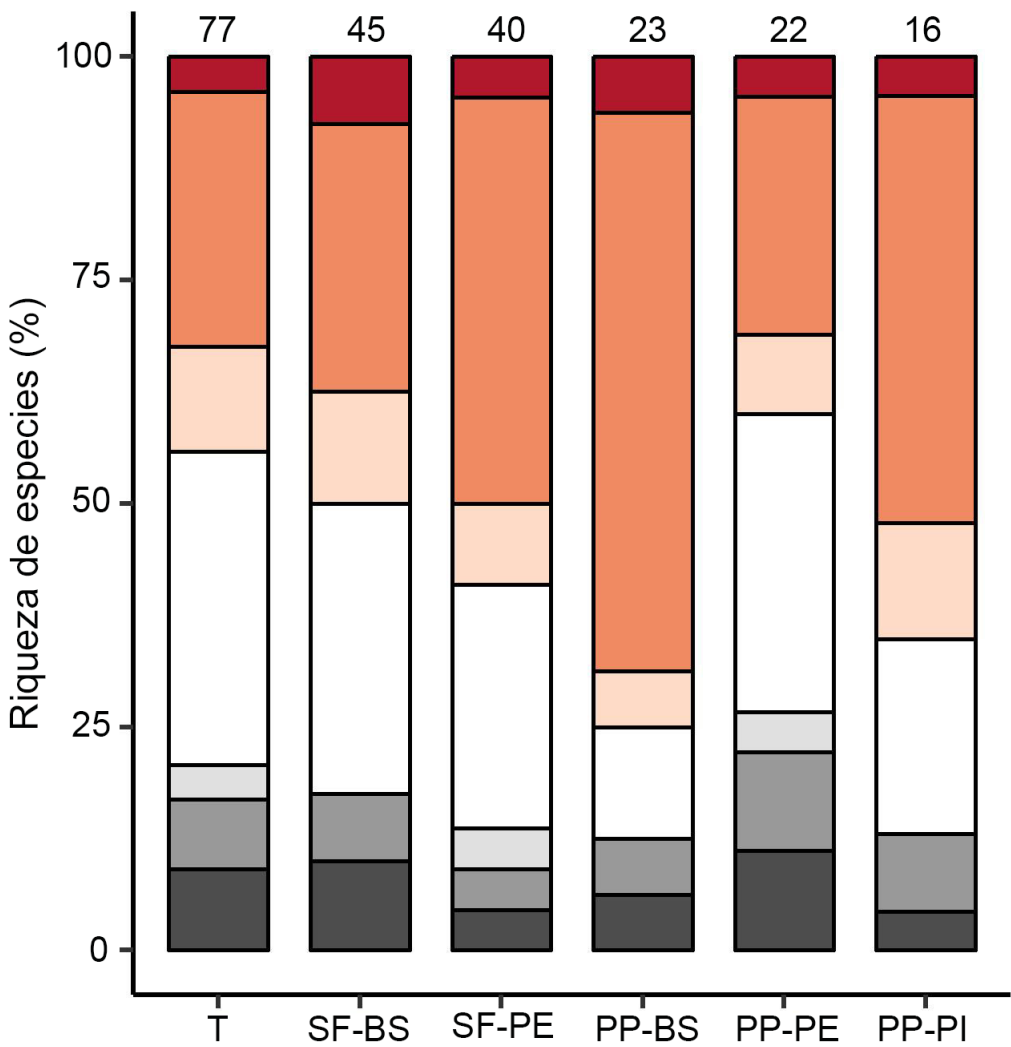

\begin{tabular}{l}
$\square$ \\
\hline$\square$ Araceae \\
Bromeliaceae \\
Cactaceae \\
\hline \hline Orchidaceae \\
Otras \\
\hline \hline Piperaceae \\
Polypodiaceae
\end{tabular}

Figura 3: Contribución de las familias a la riqueza de epífitas vasculares en 110 árboles remanentes del género Ficus L. ubicados en cinco sitios en la Depresión Central de Chiapas, México. Los valores ubicados encima de cada barra representan el número de especies. T=Total, SF-BS=San Fernandobosque semiconservado, SF-PE=San Fernando-pastoreo extensivo, PP-BS=Piedra Parada-bosque semiconservado, PP-PE=Piedra Parada-pastoreo extensivo, PP-PI=Piedra Parada-pastoreo intensivo. Otras=Araliaceae, Clusiaceae y Dryopteridaceae. 
Cuadro 1: Comparación de los atributos (promedio y desviación estándar) de las epífitas vasculares en cinco sitios ubicados en la Depresión Central de Chiapas, México. Letras diferentes ( $a, b, c)$ indican diferencias significativas (Prueba de Tukey: $p<0.05$ ). SF-BS=San Fernando-bosque semiconservado, SF-PE=San Fernando-pastoreo extensivo, PP-BS=Piedra Parada-bosque semiconservado, PP-PE=Piedra Parada-pastoreo extensivo, PP-PI=Piedra Parada-pastoreo intensivo.

\begin{tabular}{|c|c|c|c|c|c|}
\hline \multirow{2}{*}{ Variable } & \multicolumn{5}{|c|}{ Sitio } \\
\hline & SF-BS & SF-PE & PP-BS & PP-PE & PP-PI \\
\hline Abundancia & $107.6(80.8)^{\mathrm{ab}}$ & $43.3(39.4)^{c}$ & $135.7(83.0)^{\mathrm{a}}$ & $63.6(76.9)^{\mathrm{bc}}$ & $34.2(50.4)^{c}$ \\
\hline Riqueza & $9.1(4.5)^{a}$ & $5.5(2.5)^{b}$ & $6.0(2.0)^{b}$ & $4.8(1.5)^{b c}$ & $3.4(1.0)^{c}$ \\
\hline Shannon (exponencial) & $5.2(2.0)^{\mathrm{a}}$ & $3.5(1.2)^{b}$ & $3.6(0.6)^{b}$ & $3.3(1.1)^{b}$ & $2.8(1.1)^{b}$ \\
\hline
\end{tabular}

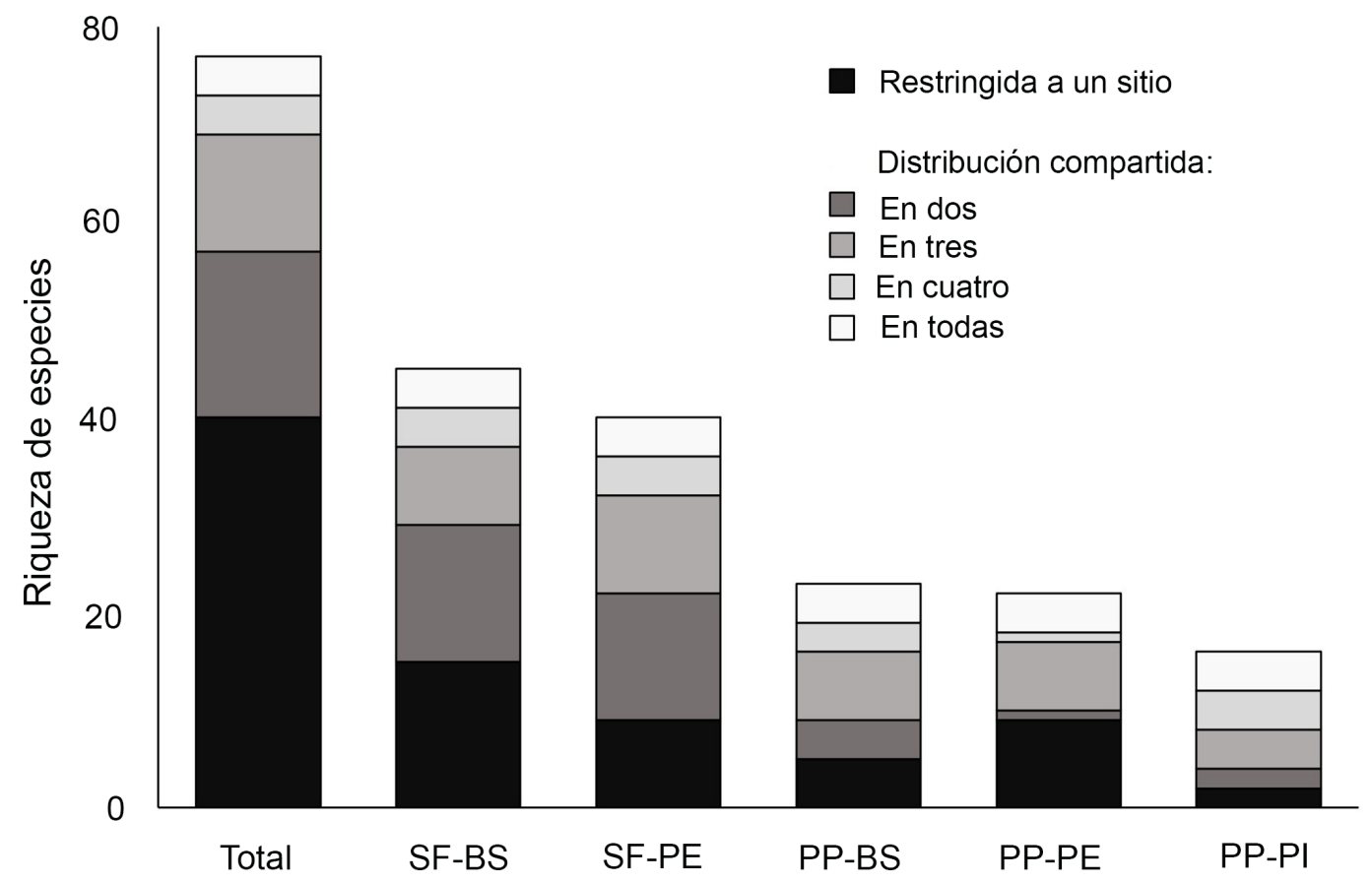

Figura 4: Especies exclusivas y compartidas de epífitas vasculares en dos o más sitios evaluados en la Depresión Central de Chiapas, México. SF$\mathrm{BS}=$ San Fernando-bosque semiconservado, SF-PE=San Fernando-pastoreo extensivo, PP-BS=Piedra Parada-bosque semiconservado, PP-PE=Piedra Parada-pastoreo extensivo, PP-PI=Piedra Parada-pastoreo intensivo.

occulta Mart.-Correa, Espejo \& López-Ferr., T. makoyana Baker y T. juncea (Ruiz \& Pav.) Poir., así como la cactácea Rhipsalis baccifera (Sol.) Stearn. En el NMDS se observa que la composición de especies es diferente entre los bosques semiconservados de ambas localidades y el SF-PE (Fig. 5). La composición de especies en los sistemas silvopastoriles presenta menor diferenciación en comparación a la observada en los bosques semiconservados de ambas localidades. La composición del PP-PI fue la más homogé- nea y contrasta con la mayor heterogeneidad detectada en los otros sitios. Se encontraron diferencias estadísticas en la composición de especies entre sitios (PERMANOVA: $R_{2}=0.19, p<0.01$ ).

Relación entre el tamaño del árbol (DAP) y la diversidad alfa de epífitas

Se encontraron diferencias significativas entre los DAP de los árboles $\left(F_{4,105}=7.692, p<0.001\right)$. La prueba de Tukey in- 


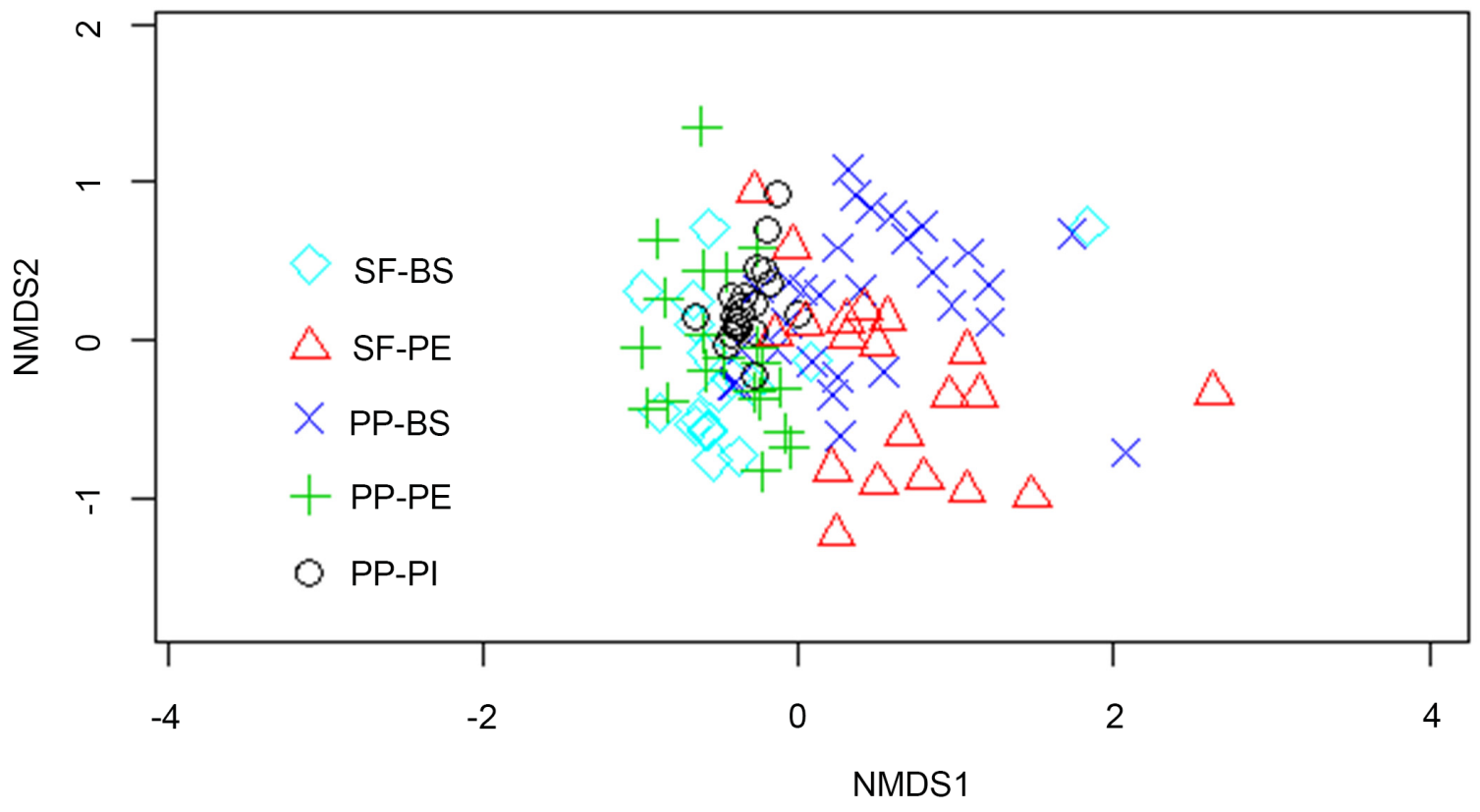

Figura 5: Análisis no métrico multidimensional (NMDS) basado en la composición de las epífitas vasculares en 110 árboles remanentes del género Ficus L. ubicados en cinco sitios en la Depresión Central de Chiapas, México. SF-BS=San Fernando-bosque semiconservado, SF-PE=San Fernando-pastoreo extensivo, PP-BS=Piedra Parada-bosque semiconservado, PP-PE=Piedra Parada-pastoreo extensivo, PP-PI=Piedra Parada-pastoreo intensivo.

dica que las diferencias en DAP son entre los sitios SF-BS vs. PP-PI, SF-BS vs. PP-BS, SF-PE vs. PP-PI y PP-PE vs. PP$\mathrm{PI}(\mathrm{p}<0.05$, en todos los casos) (Fig. 6). Los árboles de Ficus tuvieron en promedio un DAP de $58.6 \mathrm{~cm}$ (DE=29.34, Min=18.56, Max=150.88). Los árboles con DAP más grandes se encontraron en SF-BS y PP-PE; en cambio, los de menor tamaño estuvieron en PP-PI. Los modelos de regresión lineal indican que, en general, la diversidad alfa de epífitas se incrementa ligeramente y de manera positiva con un mayor tamaño en DAP de los árboles $\left(p<0.05, R^{2}{ }_{\text {adj }}=0.05\right)$. A nivel de sitio, únicamente en PP-BS se observó un aumento de la diversidad con el tamaño de los árboles $(p<0.05$, $\left.\mathrm{R}^{2}{ }_{\mathrm{adj}}=0.16\right)$.

\section{Discusión}

El enfoque de este estudio es tratar de comprender el papel de los árboles remanentes del género Ficus como refugio para albergar a las especies de epífitas en los sistemas silvopastoriles. Se observó una variación en la diversidad y composición de epífitas entre los sitios evaluados, con un incremento de la diversidad y una composición más heterogénea de las epífitas en los fragmentos de bosques semi- conservados, en comparación con los árboles remanentes dentro de los pastizales. Sin embargo, estos últimos contribuyen de manera importante a la diversidad total de epífitas.

\section{Composición florística}

La riqueza de epífitas vasculares (77 especies) encontradas en 110 árboles hospederos de tres especies de Ficus es relevante, principalmente porque el estudio se realizó en paisajes con una fuerte presión antropogénica. Esta cifra es parecida a las detectadas en otros estudios similares que se realizaron en bosques tropicales de Panamá (56 especies; Einzmann et al., 2016) y en Los Tuxtlas, México (83 especies; Hietz-Seifert et al., 1996). Esta riqueza representa $6.7 \%$ del total de epífitas vasculares estimadas para el estado de Chiapas (Wolf y Flamenco, 2003).

Las dos familias más importantes en este estudio (Orchidaceae y Bromeliaceae) también han sido las de mayor relevancia en otros trabajos parecidos al nuestro, aunque estos se han realizado en bosques húmedos de montañas (Krömer et al., 2007b; Flores-Palacios y García-Franco, 2008; Larrea y Werner, 2010; Poltz y Zotz, 2011). Destaca 


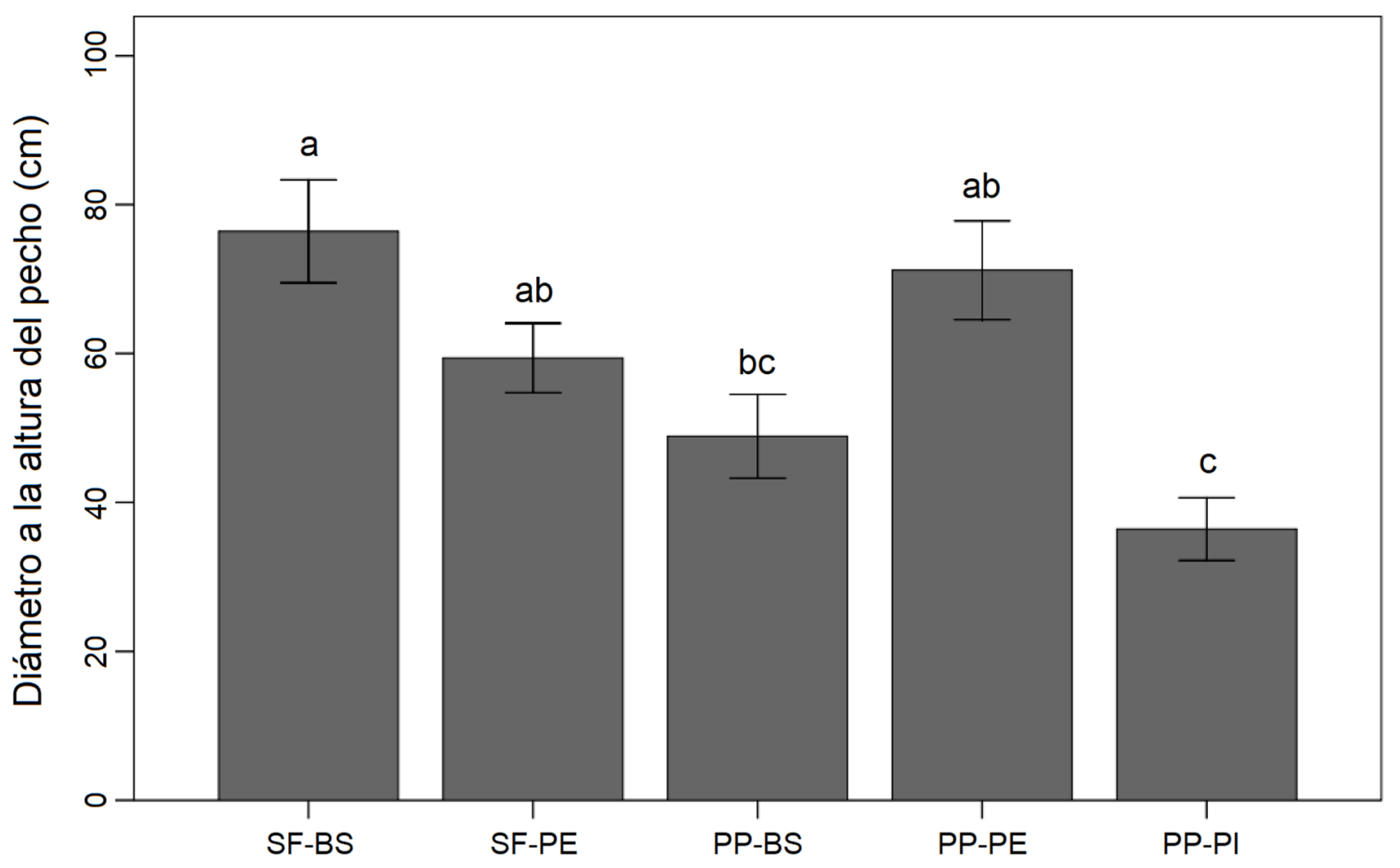

Figura 6: Valores del diámetro a la altura del pecho de los árboles remanentes del género Ficus L. en cinco sitios en la Depresión Central de Chiapas, México. Valores promedios \pm error estándar. Letras diferentes $(a, b, c)$ indican diferencia significativa (Prueba de Tukey: $p<0.05)$. SF-BS=San Fernandobosque semiconservado, SF-PE=San Fernando-pastoreo extensivo, PP-BS=Piedra Parada-bosque semiconservado, PP-PE=Piedra Parada-pastoreo extensivo, PP-PI=Piedra Parada-pastoreo intensivo.

también Cactaceae como la tercera familia con mayor riqueza, aunque esta no es relevante en los bosques húmedos en comparación con Orchidaceae y Bromeliaceae. No hay estudios que hayan demostrado hasta este momento la importancia de las cactáceas en el bosque tropical seco. Pero estos se ven favorecidos por ambientes en donde hay poca disponibilidad de agua, se presenta un periodo largo de sequía y los árboles pierden sus hojas con efectos estresantes principalmente en las especies mésicas y no tanto en las xerófitas, como las cactáceas (Andrade y Nobel, 1997; Wagner et al., 2015). Las especies epífitas de cactáceas tienen una alta capacidad de adaptación a la falta de disponibilidad de agua debido a su metabolismo ácido de las crasuláceas (CAM, por sus siglas en inglés) y a su capacidad de almacenamiento de reservas de agua y nutrientes (Andrade y Nobel, 1997). Se anticipaba la contribución a la riqueza total de la familia Bromeliaceae, ya que muchas de sus especies han desarrollado adaptaciones morfológicas y ecofisiológicas (p.ej., mayor cantidad de tricomas espe- cialmente en bromelias atmosféricas, presentan metabolismo de fotosíntesis CAM y tienen hojas arrosetadas que retienen agua en sus tanques) que les permite colonizar los bosques tropicales secos (Mondragon y Calvo-Irabien, 2006; Chilpa-Galván et al., 2013, 2017). Esto a pesar de las condiciones ambientales estresantes que imperan en estos bosques en comparación con sus contrapartes más húmedos (p.ej., sequía más severa, menor humedad y mayor radiación solar).

Las orquídeas en general son más susceptibles al disturbio antrópico (Turner et al., 1994; Krömer et al., 2014; Pérez-Peña y Krömer, 2017). No obstante, la resistencia a la sequía observada en la mayoría de las orquídeas epífitas es proporcionada por un comportamiento fotosintético CAM fuerte, que promueve una economía de agua muy favorable (Kerbauy et al., 2012). Incluso varios géneros han desarrollado mecanismos para hacer uso eficiente del agua (metabolismo CAM) y retenerla. Por ejemplo, Trichocentrum Poepp. \& Endl. es un género con metabolis- 
mo CAM (Zotz, 2004) que tiene hojas suculentas, debido a que la hipodermis funciona como almacén de agua; mientras que los pseudobulbos de algunos géneros (Oncidium Sw., Catasetum Rich. ex Kunth y Guarianthe Dressler \& W.E. Higgins) Ilegan a almacenar hasta 95\% del agua de toda la planta (Zotz y Hietz, 2001; De la Rosa-Manzano et al., 2014).

En el nivel taxonómico inferior a familia, es importante destacar la contribución del género Tillandsia (Bromeliaceae) en la riqueza (16 especies o $20 \%$ del total) y abundancia (6667 individuos o 80\% del total). También fueron de las únicas que estuvieron presentes en todos los sitios. Esto sugiere la importancia del conjunto de adaptaciones que han adquirido con relación a las condiciones secas y de disturbio (p.ej., paredes y cutículas gruesas en la epidermis que minimizan la pérdida de agua). Asimismo, el sureste de México es una zona de diversificación de este grupo (Zotz y Schultz, 2008). La baja contribución del resto de familias y en particular de las aráceas, las piperáceas y los helechos (p.ej., Polypodiaceae) se debe a que típicamente son elementos diversos en elevaciones intermedias (1000-2500 m s.n.m.) o regiones de mayor humedad, precipitación y de temperaturas más frescas a las que se presentan en nuestra área de estudio (Krömer et al., 2005; Hietz et al., 2006; Acebey y Krömer, 2008; Carvajal-Hernández y Krömer, 2015; Vergara-Rodríguez et al., 2017; Jiménez-López et al., 2020).

\section{Patrones de diversidad}

La reducción de la diversidad alfa de epífitas sugiere un efecto de la estructura del paisaje y del aislamiento de los árboles. El sitio con manejo tecnificado de los pastos y con pocos árboles (PP-PI) tuvo apenas la mitad de las especies en promedio que los sitios de bosques semiconservados. Esta evidencia es congruente con lo detectado en las familias de este gremio ecológico que generalmente reducen su diversidad con un incremento en los niveles de disturbio antrópico (Barthlott et al., 2001; Krömer et al., 2007a, 2014; Köster et al., 2009; Poltz y Zotz, 2011; Toledo-Aceves y Hernández-Apolinar, 2016). Las implicaciones que imponen los sistemas silvopastoriles en los árboles remanentes, por un efecto espacial, es que están expuestos a condiciones ambientales distintas a la de aquellos árboles dentro del bosque, principalmente a niveles elevados de radiación solar, temperatura elevada, mayor turbulencia del viento y un aumento en la tasa de evapotranspiración potencial (Laurance, 2004; Larrea y Werner, 2010). Esto limita el establecimiento y el crecimiento de muchas especies de epífitas, principalmente grupos higrófilos (vulnerables a sequía) y umbrófilos (susceptibles a la alta incidencia de luz) no tan competitivos como algunas bromelias, orquídeas y helechos xerotolerantes (Krömer et al., 2014). Por ejemplo, las bromelias y orquídeas han desarrollado mecanismos de adaptación a estas condiciones ambientales como lo mencionamos anteriormente (p.ej., tricomas o pseudobulbos para capturar o almacenar agua y CAM) (Zotz y Hietz, 2001).

La capacidad de las epífitas para explotar ciertos recursos como la intensidad de la luz y la humedad, determina en parte la composición y distribución vertical de las especies en los árboles hospederos (Krömer et al., 2007b; De la Rosa-Manzano et al., 2014). Aunque también dependen de las propiedades arquitectónicas y de las características de la corteza que estos tengan (p.ej., DAP, altura, inclinación/grosor de ramas, rugosidad y capacidad de retención de agua) (Mehltreter et al., 2005; Larrea y Werner, 2010; Dislich y Mantovani, 2016). Ya que estos presentan, en ocasiones, características únicas que determinan la calidad del hospedero para las epífitas (Vergara-Torres et al., 2010). Los árboles de Ficus muestreados, especialmente F. petiolaris y F. maxima son de corteza rugosa, lo que puede facilitar el anclaje de las epífitas. Los Ficus pueden ser buenos indicadores de cómo se dan estas modificaciones en los patrones de diversidad de epífitas al contrastar su presencia con relación a un gradiente de disturbio y de aislamiento (Gonçalves y Waechter, 2002); sin dejar de considerar que la edad y tamaño de los árboles, suelen ser determinantes también para observar un incremento en la diversidad de epífitas (Hietz y Hietz-Seifert, 1995; Flores-Palacios y García-Franco, 2008).

La composición de epífitas es afectada por el aislamiento de los árboles de Ficus y los cambios ambientales asociados a estos, lo cual promueve una mayor heterogeneidad. Es notorio el incremento en la riqueza y abundancia de las especies del género Tillandsia en los sistemas silvopastoriles, que son tolerantes a las condiciones de menor humedad y mayor incidencia solar. Este patrón ha sido documentado para otras comunidades arbóreas de mayor 
humedad sujetos a disturbio antrópico, como los bosques de montaña, en donde algunas especies de Tillandsia son claramente abundantes y dominantes (Hietz y Hietz-Seifert, 1995; Wolf, 2005; Larrea y Werner, 2010; Krömer et al., 2014).

\section{Los árboles de Ficus y conservación de epífitas}

Nuestros resultados avalan que los árboles de Ficus representan una fuente de hábitat importante para las epífitas. Además de la corteza rugosa de algunas de sus especies, también presentan una ramificación densa y gruesa, que se extienden horizontalmente, aumentando el área disponible para la colonización de epífitas (Waechter, 1992; Rudolph et al., 1998). Catchpole y Kirkpatrick (2011) mencionan que las hemiepífitas ocurren principalmente en los puntos en donde los ejes de las ramas se interceptan y que hay una relación entre la inclinación y el diámetro de las ramas, y entre la longitud y el área de la superficie que ofrece condiciones para su asentamiento. Son pocas las investigaciones que han documentado la diversidad de epífitas presentes en árboles de Ficus. Por ejemplo, Gonçalves y Waechter (2002) en la planicie costera de Rio Grande do Sul (Brasil) obtuvieron una riqueza total y promedio de epífitas por árbol (77 y 3.5, respectivamente) similar a los resultados obtenidos en este estudio; mientras que Köster et al. (2011), en bosques húmedos de Ecuador, encontraron promedios de 61.6 y 71.7 en dos localidades y Krömer et al. (2005) obtuvieron un valor máximo de 83 epífitas en un árbol de Ficus en Bolivia. Esta evidencia sugiere que los árboles remanentes de este género pueden ayudar a mantener y proveer de hábitats a las epífitas, principalmente en paisajes sujetos a una fuerte presión por las actividades humanas.

Lo anterior debe motivar acciones de concientización para promover la permanencia de árboles de Ficus dentro de los sistemas silvopastoriles, como ya se hace con Acacia pennatula, porque es utilizada como forraje para el ganado bovino en la misma zona de estudio (Ramírez-Marcial et al., 2012). Esta relación entre árbol hospedero y epífita puede coadyuvar también en la facilitación de otras interacciones ecológicas como la disponibilidad de recursos alimenticios, sitios de anidación y materiales para nidos para las aves (Cruz-Angón y Greenberg, 2005) y favorecer la conectividad ecológica y de dispersión de distintos grupos de plantas y animales en estos paisajes altamente fragmentados (SerioSilva et al., 2002; Piedra-Malagón et al., 2006).

Finalmente, en este estudio no se analizó la relación distancia del árbol remanente al fragmento de bosque semiconservado para determinar si hay un incremento de la diversidad a menor distancia al bosque. Algunos estudios sugieren que cuando los árboles remanentes se encuentran más cercanos a un área de vegetación, se favorece el tiempo de colonización de especies epífitas (Sillett et al., 1995; Hietz-Seifert et al., 1996; Einzmann et al., 2016). Sin embargo, la configuración espacial en la estructura de estos paisajes es difícil de delimitar, y no es fácil determinar cuál es el fragmento más cercano que pudiera servir como fuente de propágulos de las epífitas (semillas, esporas). Además no es posible determinar, con certeza, en el caso de los árboles remanentes dentro de los sistemas silvopastoriles, si estos crecieron posteriormente al cambio de uso de suelo, o si fueron dejados a propósito por sus bondades como sombra y alimento para el ganado vacuno. Independientemente del origen de los árboles de Ficus en los sistemas silvopastoriles, estos pueden albergar una cantidad considerable de epífitas y coadyuvan en complementar la diversidad regional del paisaje. Lo anterior es importante principalmente en regiones en donde antes predominaban los bosques tropicales secos y que están sujetos a fuertes cambios de uso de suelo, como sucede en la Depresión Central de Chiapas.

\section{Financiamiento}

Este estudio fue apoyado por la Coordinación de la Unidad Villa Corzo de la Universidad de Ciencias y Artes de Chiapas a través del proyecto "Biodiversidad y conservación en sistemas agroforestales del sureste de México" dirigido por RMC.

\section{Contribución de autores}

IATC, RMC y NMM concibieron y diseñaron el estudio. IATC y RMC coordinaron el trabajo de campo. IATC, RMC y DAJL realizaron los análisis. IATC y RMC escribieron el manuscrito con la ayuda de DAJL y NMM. Todos los autores contribuyeron a la determinación taxonómica, la interpretación de los resultados, la discusión, revisión y aprobación del manuscrito final. 


\section{Agradecimientos}

Los autores agradecen a la directiva del parque Sima de las Cotorras (San Fernando) y a Alberto Gómez, por otorgar los permisos para realizar parte del estudio en sus terrenos; a Alberto Gómez, Eli Morales Alfaro, Carlos Aguilar Castillo, Abner Mendoza Gómez, Rogelio López Pereyra, Jonathan Agustín Sánchez, Roberto García Sánchez y Jesús Arcos Álvarez quienes apoyaron en el trabajo de campo; a Roberto García Martínez, que aportó información complementaria del muestreo de epífitas en la localidad de San Fernando.

\section{Literatura citada}

Acebey, A. y T. Krömer. 2008. Diversidad y distribución de Araceae de la Reserva de la Biosfera Los Tuxtlas, Veracruz, México. Revista Mexicana de Biodiversidad 79(2): 465-471. DOI: https://doi.org/10.22201/ib.20078706e.2008.002.553

Andrade, J. L. y P. S. Nobel. 1997. Microhabitats and water relations of epiphytic cacti and ferns in a Lowland Neotropical Forest. Biotropica 29(3): 261-270. DOI: https:// doi.org/10.1111/j.1744-7429.1997.tb00427.x

Arellano, L., J. L. León-Cortés y G. Halffter. 2008. Response of dung beetle assemblages to landscape structure in remnant natural and modified habitats in southern Mexico. Insect Conservation and Diversity 1(4): 253-262. DOI: https://doi. org/10.1111/j.1752-4598.2008.00033.x

Arellano, L., J. L. León-Cortés, G. Halffter y J. Montero. 2013. Acacia woodlots, cattle and dung beetles (Coleoptera: Scarabaeinae) in a Mexican silvopastoral landscape. Revista Mexicana de Biodiversidad 84(2): 650-660. DOI: https://doi. org/10.7550/rmb.32911

Armenteras, D., J. M. Espelta, N. Rodríguez y J. Retana. 2017. Deforestation dynamics and drivers in different forest types in Latin America: Three decades of studies (1980-2010). Global Environmental Change 46: 139-147. DOI: https:// doi.org/10.1016/j.gloenvcha.2017.09.002

Barthlott, W., V. Schmit-Neuerburg, J. Nieder y S. Engwald. 2001. Diversity and abundance of vascular epiphytes: A comparison of secondary vegetation and primary montane rain forest in the Venezuelan Andes. Plant Ecology 152(2): 145-156. DOI: https://doi.org/10.1023/A:1011483901452

Benhin, J. K. A. 2006. Agriculture and deforestation in the tropics: A critical theoretical and empirical review. AMBIO: A Journal of the Human Environment 35(1): 9-16. DOI: https://doi. org/10.1579/0044-7447-35.1.9

Beutelspacher-Baigts, C. R. 2013. Guía de orquídeas de Chiapas. Consejo de Ciencia y Tecnología del Estado de Chiapas. Tuxtla Gutiérrez, México. 187 pp.

Cach-Pérez, M. J., J. L. Andrade, N. Chilpa-Galván, M. TamayoChim, R. Orellana y C. Reyes-García. 2013. Climatic and structural factors influencing epiphytic bromeliad community assemblage along a gradient of water-limited environments in the Yucatan Peninsula, Mexico. Tropical Conservation Science 6(2): 283-302. DOI: https://doi. org/10.1177/194008291300600209

Callaway, R. M., K. O. Reinhart, G. W. Moore, D. J. Moore y S. C. Pennings. 2002. Epiphyte host preferences and host traits: mechanisms for species-specific interactions. Oecologia 132(2): 221-230. DOI: https://doi.org/10.1007/s00442002-0943-3

Carvajal-Hernández, C. I. y T. Krömer. 2015. Riqueza y distribución de helechos y licófitos en el gradiente altitudinal del Cofre de Perote, centro de Veracruz, México. Botanical Sciences 93(3): 601-614. DOI: https://doi.org/10.17129/botsci.165

Catchpole, D. J. y J. B. Kirkpatrick. 2011. The outstandingly speciose epiphytic flora of a single strangler fig (Ficus crassiuscula) in a Peruvian montane cloud forest. In: Bruijnzeel, L. A., F. N. Scatena y L. S. Hamilton (eds.). Tropical montane cloud forests: Science for conservation and management, Cambridge University Press. Cambridge. Pp. 142-146. DOI: https://doi.org/10.1017/ CB09780511778384.015

Chilpa-Galván, N., G. Zotz, G. J. Sánchez-Fuente, C. EspadasManrique, J. L. Andrade y C. Reyes-García. 2017. Drought, post-dispersal seed predation, and the establishment of epiphytic bromeliads (Tillandsia spp.). Biotropica 49(6): 770-773. DOI: https://doi.org/10.1111/btp.12482

Chilpa-Galván, N., M. Tamayo-Chim, J. L. Andrade y C. ReyesGarcía. 2013. Water table depth may influence the asymmetric arrangement of epiphytic bromeliads in a tropical dry forest. Plant Ecology 214(8): 1037-1048. DOI: https://doi.org/10.1007/s11258-013-0229-3

Cruz-Angón, A. y R. Greenberg. 2005. Are epiphytes important for birds in coffee plantations? An experimental assessment. Journal of Applied Ecology 42(1): 150-159. DOI: https://doi. org/10.1111/j.1365-2664.2004.00983.x 
De la Rosa-Manzano, E., J. L. Andrade, G. Zotz y C. Reyes-García. 2014. Respuestas fisiológicas a la sequía, de cinco especies de orquídeas epífitas, en dos selvas secas de la península de Yucatán. Botanical Sciences 92(4): 607-616. DOI: https:// doi.org/10.17129/botsci.139

Dislich, R. y W. Mantovani. 2016. Vascular epiphyte assemblages in a Brazilian Atlantic Forest fragment: investigating the effect of host tree features. Plant Ecology 217(1): 1-12. DOI: https://doi.org/10.1007/s11258-015-0553-x

Einzmann, H. J. R., L. Döcke y G. Zotz. 2016. Epiphytes in human settlements in rural Panama. Plant Ecology and Diversity 9(3): 277-287. DOI: https://doi.org/10.1080/17550874.20 16.1177127

Einzmann, H. J. R., J. Beyschlag, F. Hofhans, W. Wanek y G. Zotz. 2015. Host tree phenology affects vascular epiphytes at the physiological, demographic and community level. AoB PLANTS 7: plu073. DOI: https://doi.org/10.1093/aobpla/ plu073

Flores-Palacios, A. y J. G. García-Franco. 2006. The relationship between tree size and epiphyte species richness: Testing four different hypotheses. Journal of Biogeography 33(2): 323-330. DOI: https://doi.org/10.1111/j.13652699.2005.01382.x

Flores-Palacios, A. y J. G. García-Franco. 2008. Habitat isolation changes the beta diversity of the vascular epiphyte community in lower montane forest, Veracruz, Mexico. Biodiversity and Conservation 17(1): 191-207. DOI: https:// doi.org/10.1007/s10531-007-9239-6

Galindo-González, J., S. Guevara y V. J. Sosa. 2000. Bat-and birdgenerated seed rains at isolated trees in pastures in a tropical rainforest. Conservation Biology 14(6): 1693-1703. DOI: https://doi.org/10.1111/j.1523-1739.2000.99072.x

Gentry, A. H. y C. Dodson 1987a. Diversity and biogeography of neotropical vascular epiphytes. Annals of the Missouri Botanical Garden 74(2): 205-233. DOI: https://doi. org/10.2307/2399395

Gentry, A. H. y C. Dodson. 1987b. Contribution of nontrees to species richness of a tropical rain forest. Biotropica 19(2): 149-156. DOI: https://doi.org/10.2307/2388737

Gibbs, H. K., A. S. Ruesch, F. Achard, M. K. Clayton, P. Holmgren, N. Ramankutty y J. A. Foley. 2010. Tropical forests were the primary sources of new agricultural land in the 1980s and 1990s. Proceedings of the National Academy of Sciences of the United States of America 107(38): 16732-16737. DOI: https://doi.org/10.1073/pnas.0910275107

Gonçalves, C. N. y J. L. Waechter. 2002. Epífitos vasculares sobre espécimes de Ficus organensis isoladas no norte da planície costeira do Rio Grande do Sul: Padrões de abundância e distribuição. Acta Botanica Brasilica 16(4): 429-441. DOI: https://doi.org/10.1590/S0102-33062002000400006

Gordillo-Ruiz, M. C. y M. A. Castillo-Santiago. 2017. Cambio de uso del suelo en la cuenca del río Sabinal, Chiapas, México. Ecosistemas y Recursos Agropecuarios 4(10): 39-49. DOI: https://doi.org/10.19136/era.a4n10.803

Graesser, J., T. M. Aide, H. R. Grau y N. Ramankutty. 2015. Cropland/pastureland dynamics and the slowdown of deforestation in Latin America. Environmental Research Letters 10(3): 034017. DOI: https://doi.org/10.1088/17489326/10/3/034017

Guevara, S., J. Meave, P. Moreno-Casasola y J. Laborde. 1992. Floristic composition and structure of vegetation under isolated trees in neotropical pastures. Journal of Vegetation Science 3(5): 655-664. DOI: https://doi. org/10.2307/3235833

Hágsater, E., M. Á. Soto, G. A. Salazar, R. Jiménez, M. A. López y R. L. Dressler. 2005. Las orquídeas de México. Instituto Chinoín. México, D.F., México. 304 pp.

Hietz, P. 2010. Ecology and ecophysiology of epiphytes in tropical montane cloud forests. In: Bruijnzeel, L. A., F. N. Scatena y L. S. Hamilton (eds.). Tropical montane cloud forests: Science for conservation and management. Cambridge University Press. Cambridge. Pp. 67-76. DOI: https://doi.org/10.1017/ CB09780511778384.007

Hietz, P., G. Buchberger y M. Winkler. 2006. Effect of forest disturbance on abundance and distribution of epiphytic bromeliads and orchids. Ecotropica 12: 103-112.

Hietz, P. y U. Hietz-Seifert. 1995. Structure and ecology of epiphyte communities of cloud forest in central Veracruz, Mexico. Journal of Vegetation Science 6(5): 719-728. DOI: https://doi.org/10.2307/3236443

Hietz-Seifert, U., P. HietzyS. Guevara. 1996. Epiphyte vegetation and diversity on remnant trees after forest clearance in Southern Veracruz, Mexico. Biological Conservation 75(2): 103-111. DOI: https://doi.org/10.1016/0006-3207(95)00071-2 
Jiménez-López, D. A., R. Martínez-Camilo, N. Martínez-Meléndez y M. Kessler. 2020. Diversity of epiphyte ferns along an elevational gradient in El Triunfo Biosphere Reserve, southern Mexico. Plant Ecology and Evolution 153(1): 1221. DOI: https://doi.org/10.5091/plecevo.2020.1573

Jost, L. 2006. Entropy and diversity. Oikos 113(2): 363-375. DOI: https://doi.org/10.1111/j.2006.0030-1299.14714.x

Kerbauy, G. B., C. A. Takahashi, A. M. Lopez, A. T. Matsumura, L. Hamachi, L. M. Félix, P. N. Pereira, L. Freschi y H. Mercier. 2012. Crassulacean acid metabolism in epiphytic orchids: current knowledge, future perspectives. In: Najafpour, M. M. (ed.). Applied photosynthesis, InTechOpen. Rijeka, Croacia. Pp. 81-104. DOI: https://doi.org/10.5772/29144

Köster, N., J. Nieder y W. Barthlott. 2011. Effect of host tree traits on epiphyte diversity in natural and anthropogenic habitats in Ecuador. Biotropica 43(6): 685-694. DOI: https://doi. org/10.1111/j.1744-7429.2011.00759.x

Köster, N., K. Friedrich, J. Nieder y W. Barthlott. 2009. Conservation of epiphyte diversity in an Andean landscape transformed by human land use. Conservation Biology 23(4): 911-919. DOI: https://doi.org/10.1111/j.1523-1739.2008.01164.x

Kreft, H., N. Köster, W. Küper, J. Nieder y W. Barthlott. 2004. Diversity and biogeography of vascular epiphytes in Western Amazonia, Yasuní, Ecuador. Journal of Biogeography 31(9): 1463-1476. DOI: https://doi.org/10.1111/j.13652699.2004.01083.x

Krömer, T., J. G. García-Franco y T. Toledo-Aceves. 2014. Epífitas vasculares como bioindicadores de la calidad forestal: impacto antrópico sobre su diversidad y composición. In: González-Zuarth, C. A., A. Vallarino, J. C. Pérez-Jiménez y A. M. Low-Pfeng (eds.). Bioindicadores: guardianes de nuestro futuro ambiental, Instituto Nacional de Ecología y Cambio Climático / El Colegio de la Frontera Sur. México, D.F. México. Pp. 606-623.

Krömer, T., A. Acebey, J. Kluge y M. Kessler. 2013. Effects of altitude and climate in determining elevational plant species richness patterns: A case study from Los Tuxtlas, Mexico. Flora - Morphology, Distribution, Functional Ecology of Plants 208(3): 197-210. DOI: https://doi.org/10.1016/j. flora.2013.03.003

Krömer, T., S. R. Gradstein y A. Acebey. 2007a. Diversidad y ecología de epífitas vasculares en bosques montanos primarios y secundarios de Bolivia. Ecología en Bolivia 42(1): 23-33.

Krömer, T., M. Kessler y S. R. Gradstein. 2007b. Vertical stratification of vascular epiphytes in submontane and montane forest of the Bolivian Andes: the importance of the understory. Plant Ecology 189: 261-278. DOI: https:// doi.org/10.1007/s11258-006-9182-8

Krömer, T., M. Kessler, R. S. Gradstein y A. Acebey. 2005. Diversity patterns of vascular epiphytes along an elevational gradient in the Andes. Journal of Biogeography 32(10): 1799-1809. DOI: https://doi.org/10.1111/j.1365-2699.2005.01318.x

Larrea, M. L. y F. Werner. 2010. Response of vascular epiphyte diversity to different land-use intensities in a neotropical montane wet forest. Forest Ecology and Management 260(11): 1950-1955. DOI: https://doi.org/10.1016/j. foreco.2010.08.029

Laurance, W. F. 2004. Forest-climate interactions in fragmented tropical landscapes. Philosophical Transactions of the Royal Society B: Biological Sciences 359(1443): 345-352. DOI: https://doi.org/10.1098/rstb.2003.1430

Mehltreter, K., A. Flores-Palacios y J. G. García-Franco. 2005. Host preferences of low-trunk vascular epiphytes in a cloud forest of Veracruz, Mexico. Journal of Tropical Ecology 21(6): 651660. DOI: https://doi.org/10.1017/S0266467405002683

Mendieta-Leiva, G., F. N. Ramos, J. P. C. Elias, G. Zotz, M. AcuñaTarazona, F. S. Alvim, D. F. E. Barbosa, G. A. Basílio, S. P. Batke, A. M. Benavides, C. T. Blum, C. R. Boelter, P. H. S. Brancalion, M. J. Carmona, L. P. Carvalho, E. de la RosaManzano, H. J. R. Einzmann, M. Fernández, S. G. Furtado, A. L. de Gasper, V. Guzmán-Jacob, P. Hietz, M. V. Irume, D. A. Jiménez-López, M. Kessler, H. Kreft, T. Krömer, G. M. O. Machado, N. Martínez-Meléndez, P. L. S. Martins, R. M. Mello, A. F. Mendes, L. M. Neto, S. R. Mortara, C. Nardy, R. P. Oliveira, A. C. A. Pereira, L. Pillaca, A. C. Quaresma, C. R. Quiel, E. Soto Medina, A. Taylor, M. S. Vega, K. Wagner, M. S. Werneck, F. A. Werner, J. H. D. Wolf, C. E. Zartman, D. Zuleta y B. Jiménez-Alfaro. 2020. EpIG-DB: A database of vascular epiphyte assemblages in the Neotropics. Journal of Vegetation Science 31(3): 518-528. DOI: https://doi. org/10.1111/jvs.12867

Mickel, J. T. y A. R. Smith. 2004. The Pteridophytes of Mexico. Memoirs of The New York Botanical Garden 88: 1-1054. 
Mondragon, D. y L. M. Calvo-Irabien. 2006. Seed dispersal and germination of the epiphyte Tillandsia brachycaulos (Bromeliaceae) in a tropical dry forest, Mexico. Southwestern Naturalist 51(4): 462-470. DOI: https://doi. org/10.1894/0038-4909(2006)51[462:SDAGOT]2.0.CO;2

Nieder, J., J. Prosperí y G. Michaloud. 2001. Epiphytes and their contribution to canopy diversity. Plant Ecology 153: 51-63. DOI: https://doi.org/10.1023/A:1017517119305

Oksanen, J., F. G. Blanchet, M. Friendly, R. Kindt, P. Legendre, D. McGlinn, P. R. Minchin, R. B. O’Hara, G. L. Simpson, P. Solymos, M. H. H. Stevens, E. Szoecs y H. Wagner. 2019. Vegan: Community ecology package. $\mathrm{R}$ package version 2.5 7.

Pérez-Peña, A. y T. Krömer. 2017. ¿Qué pueden aportar los acahuales y los cítricos a la conservación de las epífitas vasculares en Los Tuxtlas, Veracruz? In: Reynoso, V. H., R. I. Coates y M. L. Vázquez-Cruz (eds.). Avances y perspectivas en la investigación de los bosques tropicales y sus alrededores: La región de Los Tuxtlas, Universidad Nacional Autónoma de México. Cd. Mx., México. Pp. 569-580.

Piedra-Malagón, E. M., R. Ramírez Rodríguez y G. IbarraManríquez. 2006. El género Ficus (Moraceae) en el estado de Morelos, México. Acta Botanica Mexicana 75: 45-75. DOI: https://doi.org/10.21829/abm75.2006.1014

Pineda-Diez de Bonilla, E., J. León-Cortés y J. L. Rangel-Salazar. 2012. Diversity of bird feeding guilds in relation to habitat heterogeneity and land-use cover in a human-modified landscape in southern Mexico. Journal of Tropical Ecology 28(4): 369-376. DOI: https://doi.org/10.1017/ S026646741200034X

Poltz, K. y G. Zotz. 2011. Vascular epiphytes on isolated pasture trees along a rainfall gradient in the lowlands of Panama. Biotropica 43(2): 165-172. DOI: https://doi.org/10.1111/ j.1744-7429.2010.00669.x

R Core Team. 2020. R: A Language and Environment for Statistical Computing. R Foundation for Statistical Computing. Vienna, Austria.

Ramírez-Marcial, N., M. L. Rueda-Pérez, B. G. Ferguson y G. Jiménez-Ferrer. 2012. Caracterización del sistema agrosilvopastoril en la Depresión Central de Chiapas. Avances en Investigación Agropecuaria 16(2): 7-22.

Rocha-Loredo, A. G., N. Ramírez-Marcial y M. González-Espinosa. 2010. Riqueza y diversidad de árboles del bosque tropical caducifolio en la Depresión Central de Chiapas. Boletín de la Sociedad Botánica de México 87: 89-103. DOI: https://doi. org/10.17129/botsci.313

Rudolph D., G. Rauer, J. Nieder y W. Barthlott. 1998. Distributional patterns of epiphytes in the canopy and phorophyte characteristics in a western Andean rain forest in Ecuador. Selbyana 19(1): 27-33.

Serio-Silva, J. C., V. Rico-Gray, L. T. Hernández-Salazar y R. EspinosaGómez. 2002. The role of Ficus (Moraceae) in the diet and nutrition of a troop of Mexican howler monkeys, Alouatta palliata mexicana, released on an island in southern Veracruz, Mexico. Journal of Tropical Ecology 18(6): 913928. DOI: https://doi.org/10.1017/S0266467402002596

Sillett, S. C., S. R. Gradstein y D. I. I. Griffin. 1995. Bryophyte diversity of Ficus tree crowns from cloud forest and pasture in Costa Rica. Bryologist 98(2): 251-260. DOI: https://doi. org/10.2307/3243312

Soto-Pinto, L., G. Jiménez-Ferrer y T. Lerner-Martínez. 2008. Diseño de sistemas agroforestales para la producción y la conservación: experiencia y tradición en Chiapas. El Colegio de la Frontera Sur. San Cristóbal de Las Casas, México. 90 pp.

Taylor, A. y K. Burns. 2015. Epiphyte community development throughout tree ontogeny: an island ontogeny framework. Journal of Vegetation Science 26(5): 902-910. DOI: https:// doi.org/10.1111/jvs.12289

Toledo-Aceves, T. y M. Hernández-Apolinar. 2016. Population dynamics of the epiphytic bromeliad Tillandsia butzii in cloud forest. Acta Oecologica 71: 47-51. DOI: https://doi. org/10.1016/j.actao.2016.01.007

Turner, I. M., H. T. W. Tan, Y. C. Wee, A. B. Ibrahim, P. T. Chew y R. T. Corlett. 1994. A study of plant species extinction in Singapore: Lessons for the conservation of tropical biodiversity. Conservation Biology 8(3): 705-712. DOI: https://doi.org/10.1046/j.1523-1739.1994.08030705.x

Vaca, R. 2012. Deforestación y fragmentación de bosques secos en la Depresión Central de Chiapas, México. Tesis de doctorado. El Colegio de la Frontera Sur. San Cristóbal de Las Casas, México. 201 pp.

Vergara-Rodríguez, D., G. Mathieu, M. S. Samain, S. ArmentaMontero y T. Krömer. 2017. Diversity, distribution, and conservation status of Peperomia (Piperaceae) in the State of Veracruz, Mexico. Tropical Conservation Science 10: 1-28. DOI: https://doi.org/10.1177/1940082917702383 
Vergara-Torres, C. A., M. C. Pacheco-Álvarez y A. Flores-Palacios. 2010. Host preference and host limitation of vascular epiphytes in a tropical dry forest of central Mexico. Journal of Tropical Ecology 26(6): 563-570. DOI: https://doi. org/10.1017/S0266467410000349

Waechter, J. L. 1992. Epifitismo vascular en la llanura costera de Rio Grande do Sul. Tesis de doctorado. Universidad de São Carlos. São Carlos, Brasil. 163 pp.

Wagner, K. y G. Zotz. 2020. Including dynamics in the equation: Tree growth rates and host specificity of vascular epiphytes. Journal of Ecology 108(2): 761-773. DOI: https://doi. org/10.1111/1365-2745.13333

Wagner, K., G. Mendieta-Leiva y G. Zotz. 2015. Host specificity in vascular epiphytes: a review of methodology, empirical evidence and potential mechanisms. AoB PLANTS 7: plu092. DOI: https://doi.org/10.1093/aobpla/plu092

Werner, F. 2011. Reduced growth and survival of vascular epiphytes on isolated remnant trees in a recent tropical montane forest clear-cut. Basic and Applied Ecology 12(2): 172-181. DOI: https://doi.org/10.1016/j.baae.2010.11.002

Werner, F. y S. R. Gradstein. 2008. Seedling establishment of vascular epiphytes on isolated and enclosed forest trees in an Andean landscape, Ecuador. Biodiversity and Conservation 17(13): 3195-3207. DOI: https://doi.org/10.1007/s10531008-9421-5

Werner, F., J. Homeier y S. R. Gradstein. 2005. Diversity of vascular epiphytes on isolated remnant trees in the montane forest belt of southern Ecuador. Ecotropica 11: 21-40.

Winkler, M., K. Hülber y P. Hietz. 2005. Effect of canopy position on germination and seedling survival of epiphytic bromeliads in a Mexican humid montane forest. Annals of Botany 95(6): 1039-1047. DOI: https://doi.org/10.1093/aob/mci115

Wolf, J. H. D. 2005. The response of epiphytes to anthropogenic disturbance of pine-oak forests in the highlands of Chiapas, Mexico. Forest Ecology and Management 212(1-3): 376393. DOI: https://doi.org/10.1016/j.foreco.2005.03.027
Wolf, J. H. D. y A. Flamenco. 2003. Patterns in species richness and distribution of vascular epiphytes in Chiapas, Mexico. Journal of Biogeography 30(11): 1689-1707. DOI: https:// doi.org/10.1046/j.1365-2699.2003.00902.x

Zotz, G. 2004. How prevalent is crassulacean acid metabolism among vascular epiphytes? Oecologia 130: 184-192. DOI: https://doi.org/ 10.1007/s00442-003-1418-x

Zotz, G. 2013. The systematic distribution of vascular epiphytes-a critical update. Botanical Journal of the Linnean Society 171(3): 453-481. DOI: https://doi.org/10.1111/boj.12010

Zotz, G. 2016. Plants on plants-The biology of vascular epiphytes. Springer. Heidelberg, Alemania. 282 pp. DOI: https://doi. org/10.1007/978-3-319-39237-0_1

Zotz, G. y J. L. Andrade. 2001. Ecología de epífitas y hemiepifitas. In: Guariguata, M. y G. Kattan (eds.). Ecología de bosques Iluviosos Neotropicales, IICA. San José, Costa Rica. Pp. 271296.

Zotz, G. y P. Hietz. 2001. The physiological ecology of vascular epiphytes: Current knowledge, open questions. Journal of Experimental Botany 52(364): 2067-2078. DOI: https://doi. org/10.1093/jexbot/52.364.2067

Zotz, G. y S. Schultz. 2008. The vascular epiphytes of a lowland forest in Panama-species composition and spatial structure. Plant Ecology 195: 131-141. DOI: https://doi.org/10.1007/ s11258-007-9310-0

Zotz, G. y B. Vollrath. 2003. The epiphyte vegetation of the palm Socratea exorrhiza-correlations with tree size, tree age and bryophyte cover. Journal of Tropical Ecology 19(1): 81-90. DOI: https://doi.org/10.1017/S0266467403003092 
Apéndice. Listado de las especies de epífitas vasculares y su abundancia, en 110 árboles remanentes ubicados en cinco sitios en la Depresión Central de Chiapas, México. He=Hemiepífita, Ho=Holoepífita, SF-BS=San Fernando - bosque semiconservado, SF-PE=San Fernando-pastoreo extensivo, PPBS=Piedra Parada-bosque semiconservado, PP-PE=Piedra Parada-pastoreo extensivo, PP-PI=Piedra Parada-pastoreo intensivo.

\begin{tabular}{|c|c|c|c|c|c|}
\hline \multirow{2}{*}{ Familia/Especie } & \multicolumn{5}{|c|}{ Sitio } \\
\hline & SF-BS & SF-PF & PP-BS & PP-PE & PP-PI \\
\hline \multicolumn{6}{|l|}{ PTERIDOFITAS } \\
\hline \multicolumn{6}{|l|}{ Dryopteridaceae } \\
\hline Elaphoglossum guatemalense (Klotzsch) T. Moore, Ho & 6 & 0 & 0 & 0 & 0 \\
\hline \multicolumn{6}{|l|}{ Polypodiaceae } \\
\hline Campyloneurum angustifolium (Sw.) Fée, Ho & 1 & 6 & 0 & 0 & 0 \\
\hline Polypodium furfuraceum Schltdl. \& Cham., Ho & 22 & 0 & 0 & 0 & 0 \\
\hline Polypodium plebeium Schltdl. \& Cham., Ho & 0 & 0 & 0 & 1 & 0 \\
\hline Polypodium pleurosorum Kunze ex Mett., Ho & 26 & 0 & 0 & 0 & 0 \\
\hline Polypodium thyssanolepis A. Braun ex Klotzsch, Ho & 15 & 6 & 2 & 0 & 0 \\
\hline Polypodium sp. 1, Ho & 21 & 5 & 0 & 0 & 0 \\
\hline Polypodium sp. 2, Ho & 0 & 2 & 0 & 0 & 4 \\
\hline \multicolumn{6}{|l|}{ ANGIOSPERMAS } \\
\hline \multicolumn{6}{|l|}{ Magnólidas } \\
\hline \multicolumn{6}{|l|}{ Piperaceae } \\
\hline Peperomia deppeana Schltdl. \& Cham., Ho & 37 & 53 & 0 & 0 & 10 \\
\hline Peperomia obtusifolia (L.) A. Dietr., Ho & 2 & 2 & 0 & 0 & 0 \\
\hline Peperomia pereskiifolia (Jacq.) Kunth, Ho & 1 & 0 & 11 & 1 & 0 \\
\hline Peperomia tetraphylla Hook. \& Arn., Ho & 4 & 0 & 0 & 0 & 0 \\
\hline Peperomia tlapacoyoensis C. DC., Ho & 118 & 2 & 0 & 0 & 0 \\
\hline Peperomia sp. 1, Ho & 0 & 0 & 1 & 0 & 0 \\
\hline \multicolumn{6}{|l|}{ Monocotiledóneas } \\
\hline \multicolumn{6}{|l|}{ Araceae } \\
\hline Anthurium scandens (Aubl.) Engl., Ho & 29 & 12 & 0 & 0 & 2 \\
\hline Anthurium schlechtendalii Kunth, Ho & 5 & 12 & 0 & 0 & 0 \\
\hline Philodendron radiatum Schott, He & 0 & 3 & 1 & 1 & 0 \\
\hline \multicolumn{6}{|l|}{ Bromeliaceae } \\
\hline Aechmea bracteata (Sw.) Griseb., Ho & 3 & 0 & 0 & 0 & 0 \\
\hline Aechmea lueddemanniana (K. Koch) Brongn. ex Mez, Ho & 1 & 7 & 0 & 0 & 0 \\
\hline Billbergia pallidiflora Liebm., Ho & 0 & 0 & 0 & 8 & 0 \\
\hline Billbergia sp. 1, Ho & 0 & 0 & 0 & 1 & 0 \\
\hline Catopsis nutans (Sw.) Griseb., Ho & 8 & 0 & 2 & 0 & 0 \\
\hline Catopsis occulta Mart.-Correa, Espejo \& López-Ferr., Ho & 123 & 118 & 1 & 0 & 18 \\
\hline Tillandsia balbisiana Schult. f., Ho & 0 & 0 & 7 & 0 & 0 \\
\hline Tillandsia belloensis W. Weber, Ho & 253 & 34 & 705 & 0 & 0 \\
\hline Tillandsia caput-medusae E. Morren, Ho & 0 & 0 & 36 & 32 & 8 \\
\hline Tillandsia compressa Bertero ex Schult. \& Schult. f., Ho & 0 & 0 & 0 & 31 & 0 \\
\hline Tillandsia dasyliriifolia Baker, Ho & 75 & 0 & 0 & 0 & 0 \\
\hline Tillandsia fasciculata Sw., Ho & 173 & 18 & 8 & 154 & 27 \\
\hline Tillandsia flabellata Baker, Ho & 0 & 12 & 0 & 0 & 0 \\
\hline
\end{tabular}


Apéndice. Continuación.

\begin{tabular}{|c|c|c|c|c|c|}
\hline \multirow{2}{*}{ Familia/Especie } & \multicolumn{5}{|c|}{ Sitio } \\
\hline & SF-BS & SF-PF & PP-BS & PP-PE & PP-PI \\
\hline Tillandsia juncea (Ruiz \& Pav.) Poir., Ho & 245 & 52 & 3 & 0 & 2 \\
\hline Tillandsia maculata Ruiz \& Pav., Ho & 0 & 10 & 0 & 0 & 3 \\
\hline Tillandsia makoyana Baker, Ho & 33 & 23 & 0 & 64 & 6 \\
\hline Tillandsia recurvata (L.) L., Ho & 171 & 3 & 346 & 235 & 92 \\
\hline Tillandsia schiedeana Steud., Ho & 257 & 99 & 549 & 231 & 76 \\
\hline Tillandsia streptophylla Scheidw. ex C. Morren, Ho & 0 & 0 & 2 & 1 & 0 \\
\hline Tillandsia usneoides (L.) L., Ho & 0 & 1 & 0 & 0 & 0 \\
\hline Tillandsia zoquensis Ehlers, Ho & 144 & 636 & 929 & 449 & 431 \\
\hline Tillandsia sp. 1, Ho & 0 & 0 & 0 & 0 & 1 \\
\hline \multicolumn{6}{|l|}{ Orchidaceae } \\
\hline Catasetum integerrimum Hook., Ho & 0 & 0 & 3 & 0 & 0 \\
\hline Domingoa purpurea (Lindl.) Van den Berg \& Soto Arenas, Ho & 0 & 0 & 0 & 0 & 1 \\
\hline Epidendrum cardiophorum Schltr., Ho & 26 & 5 & 0 & 26 & 0 \\
\hline Epidendrum ciliare L., Ho & 1 & 10 & 0 & 0 & 0 \\
\hline Epidendrum martinezii L. Sánchez \& Carnevali, Ho & 0 & 0 & 2 & 0 & 0 \\
\hline Epidendrum melistagum Hágsater, Ho & 0 & 0 & 0 & 12 & 0 \\
\hline Guarianthe aurantiaca (Bateman ex Lindl.) Dressler \& W.E. Higgins, Ho & 4 & 7 & 0 & 0 & 0 \\
\hline Isochilus latibracteatus A. Rich. \& Galeotti, Ho & 1 & 0 & 0 & 0 & 0 \\
\hline Maxillaria variabilis Bateman ex Lindl., Ho & 6 & 9 & 1 & 0 & 0 \\
\hline Nemaconia striata (Lindl.) Van den Berg, Salazar \& Soto Arenas, Ho & 2 & 1 & 0 & 0 & 0 \\
\hline Nidema boothii (Lindl.) Schltr., Ho & 0 & 9 & 0 & 0 & 0 \\
\hline Notylia orbicularis A. Rich. \& Galeotti, Ho & 0 & 3 & 0 & 0 & 0 \\
\hline Oncidium leucochilum Bateman ex Lindl., Ho & 2 & 0 & 0 & 0 & 0 \\
\hline Oncidium sphacelatum Lindl., Ho & 2 & 0 & 0 & 0 & 0 \\
\hline Oncidium sp. 1, Ho & 0 & 0 & 0 & 1 & 0 \\
\hline Platystele stenostachya (Rchb. f.) Garay, Ho & 6 & 25 & 0 & 0 & 0 \\
\hline Polystachya cerea Lindl., Ho & 0 & 2 & 0 & 0 & 0 \\
\hline Prosthechea ochracea (Lindl.) W.E. Higgins, Ho & 2 & 0 & 0 & 0 & 0 \\
\hline Rhyncholaelia glauca (Lindl.) Schltr., Ho & 0 & 1 & 0 & 0 & 0 \\
\hline Scaphyglottis fasciculata Hook., Ho & 58 & 69 & 0 & 1 & 0 \\
\hline Specklinia tribuloides (Sw.) Pridgeon \& M.W. Chase, Ho & 10 & 0 & 0 & 0 & 0 \\
\hline Stelis purpurascens A. Rich. \& Galeotti, Ho & 86 & 0 & 0 & 0 & 0 \\
\hline Stelis sp. 1, Ho & 0 & 4 & 0 & 0 & 0 \\
\hline Trichocentrum andreanum (Cogn.) R. Jiménez \& Carnevali, Ho & 0 & 14 & 0 & 11 & 2 \\
\hline Trichocentrum ascendens (Lindl.) M.W. Chase \& N.H. Williams, Ho & 0 & 0 & 0 & 2 & 0 \\
\hline Trichocentrum undulatum (Sw.) Ackerman \& M.W. Chase, Ho & 66 & 0 & 1 & 0 & 0 \\
\hline Trichocentrum sp. 1, Ho & 47 & 0 & 24 & 0 & 0 \\
\hline \multicolumn{6}{|l|}{ Eudicotiledóneas } \\
\hline \multicolumn{6}{|l|}{ Araliaceae } \\
\hline Oreopanax guatemalensis (Lem. ex Bosse) Decne. \& Planch., He & 1 & 0 & 0 & 0 & 0 \\
\hline
\end{tabular}


Apéndice. Continuación.

\begin{tabular}{|c|c|c|c|c|c|}
\hline \multirow{2}{*}{ Familia/Especie } & \multicolumn{5}{|c|}{ Sitio } \\
\hline & SF-BS & SF-PF & PP-BS & PP-PE & PP-PI \\
\hline \multicolumn{6}{|l|}{ Cactaceae } \\
\hline Disocactus sp. 1, Ho & 0 & 0 & 3 & 0 & 0 \\
\hline Epiphyllum phyllanthus (L.) Haw., Ho & 8 & 0 & 0 & 0 & 0 \\
\hline Epiphyllum pumilum Britton \& Rose, Ho & 0 & 5 & 0 & 0 & 0 \\
\hline Pseudorhipsalis ramulosa (Salm-Dyck) Barthlott, Ho & 0 & 1 & 0 & 0 & 0 \\
\hline Rhipsalis baccifera (Sol.) Stearn, Ho & 37 & 15 & 75 & 0 & 1 \\
\hline Selenicereus aff. nelsonii (Weing.) Britton \& Rose, Ho & 8 & 0 & 0 & 0 & 0 \\
\hline Selenicereus grandiflorus (L.) Britton \& Rose, Ho & 6 & 1 & 0 & 0 & 0 \\
\hline Selenicereus undatus (Haw.) D.R. Hunt, Ho & 0 & 1 & 1 & 3 & 0 \\
\hline Stenocereus sp. 1, Ho & 0 & 0 & 0 & 3 & 0 \\
\hline \multicolumn{6}{|l|}{ Clusiaceae } \\
\hline Clusia sp. 1, He & 0 & 0 & 0 & 3 & 0 \\
\hline
\end{tabular}

